\title{
The Benefits of Diverse Preferences in Library Consortia*
}

\author{
Doh-Shin Jeon ${ }^{\dagger}$ and Domenico Menicucci ${ }^{\ddagger}$
}

December 21, 2015

\begin{abstract}
We study when a library consortium increases the aggregate payoff of the member libraries. We find that libraries with similar (diverse) preferences are likely to lose (gain) from building a consortium. Combining libraries with diverse preferences intensifies competition among publishers for scarce combined budgets because the asymmetry among the values of their bundles of journals is reduced. Our results suggest a strong tension between a short-term strategy and a long-term strategy as long as the former dictates forming a consortium among libraries with homogenous preferences in order to gain from quantity discounts. This tension might create a "library consortium trap". Our insight can be applied to other buyer groups as long as competition among sellers is generated by buyers' budget constraints.
\end{abstract}

Keywords: Buyer Group, Budget Constraint, Library Consortium, Academic Journals, Personalized Prices, Multimarket Contact

JEL numbers: D43, L13, L41, L86

*We thank Jay Pil Choi, Matthew Ellman, Christiaan Hogendorn, Tobias Klein, Martin Peitz, John Thanassoulis, Katie Yewell, the editor and three anonymous referees for helpful comments and the audience who attended our presentations at EARIE (Porto, 2013), ICT Conference (Paris, 2014) and Postal Economics Conference on "E-commerce, Digital Economy and Delivery Services" (Toulouse, 2014).

†Toulouse School of Economics (GREMAQ, IDEI) and CEPR. dohshin.jeon@gmail.com

‡Università degli Studi di Firenze, Italy. domenico.menicucci@dmd.unifi.it 


\section{Introduction}

The Internet and electronic publishing have brought fundamental changes into the market for academic journals. On the one hand, it allowed large publishers to practice 'Big Deal'1 pricing by bundling a large collection of journals. On the other hand, it induced libraries to form consortia, whereby libraries of a given geographical area join forces in order to share acquisition of electronic academic journals licensed through the Big Deal. Virtually every country or region has built or has the possibility of building a national or regional library consortium. North American examples include OhioLINK, the Triangle Research Libraries Network of North Carolina (TRLN), the Greater Western Library Alliance (GWLA), the Colorado Alliance of Research Libraries (CARL) and the Ontario Council of University Libraries (OCUL). Some well-known European groups include HEAL-LINK (Greek academic libraries including the National Library) and CBUC (academic libraries of Catalonia in Spain). ${ }^{2}$

In this paper, we aim to identify the strategies that make a library consortium successful from a long term point of view as suggested by Thomas A. Peters (2001a), director of Center for Library Initiatives,

"One challenge for academic library consortia is to shift gears and engage in more deliberate strategic planning with an eye to positive long-terms outcomes".

There are two kinds of "conventional wisdom" regarding library consortia. First, it is frequently argued that because of economies of scale, the more participants there are, the better the deal is (Okerson, 2015). Second, it has also been suggested that libraries organize themselves into groups of homogenous institutions with similar missions (Davis, 2002). In this paper, we focus on how a consortium affects competition among publishers and obtain results that challenge the conventional wisdom. Namely, we show that libraries can gain or lose from building a consortium and that they are more likely to gain (respectively, to lose) when the consortium is formed by libraries of diverse preferences (respectively, similar preferences).

One natural argument in favor of the economies of scale is that the buying power of a consortium increases with the number of the participants. The buying power can increase because the consortium improves either the direct bargaining power or the outside option. ${ }^{3}$ Furthermore, if competing researchers in the same field bargained together and all faced the same inconvenience together if the bargaining with a publisher broke down,

\footnotetext{
${ }^{1}$ Big Deal is defined as "any online aggregation of e-content that a publisher, aggregator, or vendor offers for sale or lease at prices and/or terms that substantially encourage acquisition of the entire corpus" (Peters, 2001b).

${ }^{2}$ Other examples include: CAUL CEIRC (Australia), ANSF (Brazil), CALIS (China), MALMAD (Israel), INFER (Italy), KESLI (Korea).

${ }^{3}$ For instance, the outside option of a consortium can increase with the number of its members if there
} 
this would improve the threat point because the scholars would not be suffering too much of a relative disadvantage. This logic would provide a rationale for building a consortium with homogenous libraries. ${ }^{4}$ However, Dewatripont et al. (2006) provide reasons for which libraries cannot improve their bargaining power much by building a consortium. ${ }^{5}$

Another argument in favor of the conventional wisdom is based on quantity discounts. If publishers propose non-linear tariffs as functions of usage, they are likely to provide quantity discounts. Then, it might be desirable to build a consortium with a large number of libraries with similar preferences. This is especially true for printed materials which are owned by the libraries on a permanent basis once they are purchased. However, the reasoning fails to apply to electronic academic journals, which are licensed on an annual basis. As publishers' ability to commit to future prices is limited, the gain from quantity discounts may last only for the short run. ${ }^{6}$

In this paper, we focus on one important aspect affecting the profitability of a consortium which has been neglected up to now. Precisely, we take a long-term view in the sense that publishers can change their prices after a consortium is formed and focus on how a consortium affects price competition among publishers. For this purpose, we build on the framework of our previous papers (Jeon and Menicucci, 2006 and 2011) where each (for-profit) publisher competes by offering its bundle of journals at a personalized price $^{7}$ to each different library (or a consortium) under complete information ${ }^{8}$ about the library's preferences and budget. ${ }^{9}$ We assume that all the budgets of the member libraries are pooled and that the consortium maximizes the joint payoff of all member libraries. In this setting, we study the conditions under which building a consortium is beneficial.

Since we consider that publishers make price offers before libraries make purchase decisions and that the outside option of each library (and a consortium) is normalized at

is an economy of scale in the benefit from sharing non-overlapping resources.

${ }^{4} \mathrm{We}$ are grateful to a referee for providing us with this argument.

${ }^{5}$ First, they argue that "since researchers do not see the various publishers as good substitutes and need access to all journals, consortia only introduce a relatively weak 'buyer power' (p.8)." Second, according to them, "this 'buyer concentration' remains however modest in comparison with publisher concentration: the largest library consortium represents 2 or $3 \%$ of global journal purchases, while the largest publisher represents more than $20 \%$ of journal sales (p.8)".

${ }^{6}$ For instance, Dewatripont et al. (2006) argue that "we may fear that consortia in fact strengthen the possibility for publishers to charge a high price for their electronic collection (p.52)."

${ }^{7}$ Actually, publishers' price offers are tailored directly to individual characteristics of libraries or consortia. According to Edlin and Rubinfeld (2004), "Here, the price that a buyer is quoted depends upon the buyer's observable characteristics."

${ }^{8} \mathrm{It}$ is now easy to estimate the value of a given journal to a library since the publisher and the library can observe the number of downloads of the journal (Gatten and Sanville, 2004 and Scigliano 2010). According to Derk Haank (2001), CEO of Elsevier Science, "What we are basically doing is to say that you pay depending on how useful the publication is for you - estimated by how often you use it."

${ }^{9}$ Jeon and Menicucci (2006) show that bundling is a dominant strategy in this setting. 
zero, our model does not capture any gain in buyer power. This implies that in our model, there is no gain from building a consortium in the case of a monopolist publisher. Therefore, we essentially focus on how a consortium affects the competition among publishers, which we think provides a long-term perspective since only competition can restrain the amount of surplus that each publisher can extract in the long run. We find that building a consortium increases the aggregate payoff of the member libraries only if their preferences are diverse enough to create a level-playing field among different individually-preferred publishers.

Since we assume that the value that a library obtains from a publisher's bundle is independent from the value that it obtains from another's bundle, competition among publishers is generated by the library's budget constraint. ${ }^{10}$ Consider competition between two bundles in the market of a given library. As the asymmetry between the two bundles' values increases above the library's budget, the library ends up consuming only its preferred bundle since there is no budget left for the other bundle. Combining libraries with negatively correlated values to form a consortium intensifies the competition between the two publishers for scarce combined budgets because the asymmetry between the two bundles' values is reduced, which increases the chance for the consortium to consume both bundles.

In order to provide a further intuition, consider the extreme case of perfectly negative correlation. Suppose that Library 1 (2) likes bundle A (B) so much that Library 1 (2) consumes only bundle A (B) in the absence of the consortium. Then, building a consortium creates a level-playing field between the two publishers such that no publisher monopolizes the market for the consortium and the consortium consumes both bundles. Conversely, in the case of two identical libraries (i.e., perfectly positive correlation), the consortium has no impact on the libraries' payoffs nor on the publishers' payoffs, since each library continues to consume the same bundles and bear the same expenses, as without the consortium. Last, consider the intermediate case in which Library 1 consumes only bundle A while Library 2 consumes both bundles A and B without the consortium. Then, the consortium can either increase or decrease the aggregate payoff of the libraries depending on the size of its budget. If the budget is small enough, publisher A can export its residual market power from Library 1 to Library 2 and monopolize the entire market of the consortium. This logic is similar to the one that multimarket contact allows firms to export residual collusive power from one market to another (Bernheim and Whinston, 1990).

In Section 5, we show that our main insight is robust to making the budget of each library endogenous. We consider that a funding agency decides whether or not to form

\footnotetext{
${ }^{10}$ In Section 7.1, we consider the case in which two bundles of journals can be either complements or substitutes and show that the main result is robust to this extension.
} 
a consortium and the budget of the consortium or each individual library. Then, our prediction for the case of positive correlation becomes sharper, as two libraries never gain from forming a consortium no matter what the degree of positive correlation. In the case of negative correlation, the result from the case of endogenous budget is remarkably similar to that from the case of exogenous budget: in both scenarios, the range of parameters for which building a consortium is beneficial increases with the absolute degree of correlation.

In Section 6, we consider two different scenarios in which each publisher can commit to a price that it will charge to the consortium before a funding agency decides whether or not to form it. In the first scenario, each publisher offers its price for the consortium while assuming that if the consortium is not formed, the publishers will compete in the market of each individual library. The latter determines a reservation utility for each library, which constrains the price that each publisher can charge to the consortium. We find that there exists an equilibrium in which each library at least weakly gains from building a consortium for all parameter values. This commitment case may correspond to a short-term situation. However, in the long run, publishers may not be constrained any more by such reservation utility. For this purpose, we consider a second scenario in which publishers can provide a menu of prices for each library and for the consortium before the funding agency decides to form a consortium. We find that the outcome of this menu pricing game is the same as that of the game in which publishers compete after the consortium is formed. As the publishers can threaten to charge high prices if the consortium is not formed, they are not constrained by the possibility for the libraries to make individual purchases. Therefore, a short-term gain may be associated with a long-term loss, generating a consortium trap.

Even through we focused on the market for academic journals, our results can be applied to other markets as long as competition among sellers is generated by buyers' budget constraints. For instance, in the market for drugs or vaccines, hospitals often form a group for joint purchase and pharmaceutical firms offer personalized non-linear tariffs. More precisely, in the French antitrust case against GlaxoSmithKline France (Autorité de Concurrence, 2007), GlaxoSmithKline offered different non-linear prices to different hospitals or groups of hospitals. French competition authority condemned the firm's practice of bundling two different drugs by arguing that because of the budget constraint of hospitals, bundling a monopoly drug with another can allow the firm to leverage the monopoly power from the former to the latter even if the two drugs are very different.

More broadly, our paper is relevant to the literature on internal capital markets (Cestone and Fumagalli, 2005, Fluck and Lynch, 1999, Gertner, Scharfstein and Stein, 1994, Scharfstein and Stein, 2000, Stein, 1997). The literature studies pros and cons of combining several projects under one conglomerate instead of having them as stand-alone firms. It focuses on financing investment projects in the presence of agency problems and 
compares centralized management of credit by a headquarters with decentralized management. Although we focus on a different aspect of input purchase, our theory offers an insight relevant to the literature as we analyze benefits and costs of central allocation of budget with respect to decentralized allocation.

The paper is organized as follows. Section 1.1 reviews the related literature. Section 2 presents our model. Section 3 obtains a general result in the case of a consortium of $n$ libraries. Section 4 provides more precise results focusing on correlation of preferences by considering a consortium of two libraries. Section 5 studies the case of endogenous budget. Section 6 studies commitment to consortium pricing. In Section 7, we briefly discuss two extensions that show the robustness of our results: Section 7.1 discusses when two bundles are substitutes or complements and Section 7.2 discusses when there are three competing publishers. The detailed analysis of the two extensions is provided in Appendix B of Supporting Information. Section 8 provides the conclusion.

\subsection{Literature review}

Our paper is related to the literature on buyer group which studies when buyers gain from forming a buyer group, more specifically to the papers that analyze the question in a framework of competing sellers: Inderst and Shaffer (2007), Marvel and Yang (2008), Dana (2012), Chen and Li (2013). Among those paper, our paper is more closely related to Inderst and Shaffer (2007) and Dana (2012) since they assume that sellers have complete information on buyers' preferences and hence can offer personalized tariffs, regardless of whether or not buyers form a group. Although the two papers differ in the way they generalize their results, ${ }^{11}$ the main insight can be obtained by considering a two-sellertwo-buyer setting in which each buyer buys one unit from only one of the two sellers. They assume that the buyer group makes exclusive purchase commitment and that sellers make a take-it-or-leave-it offer. In this setup, a buyer group never decreases the aggregate payoff of its members: it strictly increases the aggregate payoff unless they have identical preferences. Our paper differs from Inderst and Shaffer (2007) and Dana (2012) in two important assumptions: sellers compete for a limited budget and neither individual buyer nor the buyer group makes exclusive purchase commitment. This leads to a different conclusion that a buyer group can either increase or reduce the aggregate payoff of the buyers.

Our companion paper (Jeon and Menicucci, 2014) also studies the question of how a

\footnotetext{
${ }^{11}$ Inderst and Shaffer (2007) consider competition in non-linear tariff between two sellers and extend their result to a bargaining setup. They also make each seller's choice of product characteristics endogenous. Dana (2012) considers $n$ sellers, a continuum of buyers, and allows different buyer groups to get formed. He proves that the grand coalition is a coalition-proof subgame perfect equilibrium when there are two sellers.
} 
buyer group affects the aggregate payoff when sellers compete. As in the current paper, buyers do not commit to exclusive purchase. However, there are some important differences: buyers have no budget constraint and sellers sell strict substitutes and compete by offering personalized non-linear tariffs. In that paper, we focus on the equilibrium which is Pareto-dominant in terms of the sellers' payoffs and show that buyer group strictly reduces the aggregate payoff of the buyers if sellers' costs are convex but does not affect it if sellers' costs are linear or concave.

Our current paper builds on the theoretical literature on the market for academic journals which studies issues raised by the move to electronic publishing. ${ }^{12}$ The literature has studied bundling and/or price discrimination (McCabe, 2004, Jeon and Menicucci, 2006, Armstrong 2010), interoperability (Jeon and Menicucci, 2011), and open access journals (McCabe and Snyder, 2007, Jeon and Rochet, 2010, Armstrong 2014). We contribute to the literature by studying the issue of library consortia.

Although we consider a common agency under complete information (Bernheim and Whinston, 1986, 1998, Jeon and Menicucci, 2012), the well-known result that competition among sellers achieves the outcome that maximizes the joint payoff of all sellers and the buyer fails to hold in our setting because of the budget constraint. In contrast, Jeon and Menicucci (2012) show that the result holds if the common agency faces a slot constraint instead of a budget constraint.

\section{A simple model of the market for academic jour- nals}

As we mentioned in the introduction, we build on the model of our previous papers (Jeon and Menicucci, 2006 and 2011), and accordingly assume that publishers have complete information about the value that a library (or a consortium of libraries) attaches to each bundle of journals and about the library's budget (or the consortium's budget), and offer personalized prices based on that information. ${ }^{13}$

There are two (for-profit) publishers, $A$ and $B$, and $n \geq 2$ libraries; in Section 7.2, we extend the model to three publishers. We distinguish a mandatory consortium from a voluntary consortium. In the case of a mandatory consortium, a funding agency decides whether the purchase of the publishers' journals will be done in a decentralized manner (through each library) or in a centralized manner (through a library consortium). Hence,

\footnotetext{
${ }^{12}$ See Bergstrom (2001) and Dewatripont et al. (2006) for an introduction.

${ }^{13}$ In reality, some degree of asymmetric information between publishers and libraries may be present (as mentioned by one referee), but we believe that the benefit of additional insights from explicitly modelling asymmetric information is lower than the cost of making the model more complex and less tractable.
} 
once it decides to form a consortium, each library $i$ 's participation, for $i=1, \ldots, n$, is mandatory. In contrast, in the case of a voluntary consortium, the libraries collectively decide whether to form a consortium or not and therefore the participation is voluntary. In this case, the consortium's budget is determined by the contribution of each participating library. ${ }^{14}$ We mainly focus on the mandatory consortium and discuss how our analysis can be applied to the voluntary consortium.

Given that each publisher offers personalized prices, without loss of generality, we assume that each publisher offers a pure bundle of all its journals to each library or the consortium. ${ }^{15}$ Hence, publisher $j(=A, B)$ offers bundle $j$. Let $U_{j}^{i}(>0)$ denote the monetary utility that library $i(=1, \ldots, n)$ obtains from consuming bundle $j$. It is assumed to be independent of whether the library also consumes bundle $k(\neq j)$ with $k=A, B$; in Section 7.1, we consider the case in which the bundles are substitutes or complements and show the robustness of the results. Let $M^{i}(>0)$ denote the budget of library $i$. In the baseline model, the budget of each library is exogenously given whereas in Section 5 , the budget of each library is endogenously determined. The payoff of a library is equal to the utility it obtains from the bundles of journals it buys plus the budget left after the purchase. The library's utility from the remaining budget can be interpreted as the utility from using it to purchase monographs, database and journals of minor publishers.

Let $C$ represent the consortium of the $n$ libraries. As the consortium is assumed to maximize the sum of all member libraries' payoffs, the utility it obtains from consuming bundle $j$ is given by:

$$
U_{j}^{C}=\sum_{i=1}^{n} U_{j}^{i} .
$$

In the baseline model, we compare the decentralized purchase to the centralized one for a given aggregate budget. Therefore, the budget of the consortium is given by:

$$
M^{C}=\sum_{i=1}^{n} M^{i} .
$$

The payoff of the consortium is equal to the utility it obtains from the bundles it buys plus the budget left after the purchase.

Let $P_{j}^{i}(>0)$ represent the price that publisher $j(=A, B)$ charges to library $i$ (= $1, \ldots, n, C)$ for bundle $j$. We assume that the fixed cost of producing the first copy of

\footnotetext{
${ }^{14}$ For instance, according to Sloan (2000), with the Illinois Digital Academic Library (IDAL), databases are supported centrally with funds from the Illinois Board of Higher Education and the decision making is centralized. In contrast, with Illinois Bibliographic Information Service (IBIS) of Illinois Library Computer System Organization (ILCSO), database are licensed with local library funds and much decision-making rests in the hands of local libraries.

${ }^{15}$ Arguing as in the proof of Proposition 2(i) in Jeon and Menicucci (2006), we can prove that, for each publisher, pure bundling of its journals weakly dominates any alternative to pure bundling.
} 
bundle $j$ has already been incurred and that the marginal cost of distributing a journal is zero. Therefore, publisher $j$ 's profit is equal to publisher $j$ 's revenue, and social welfare is maximized when all libraries consume both bundles.

We consider a game with the following timing:

- Stage 0: The funding agency decides whether to form a consortium or not.

- Stage 1: Each publisher $j$ simultaneously chooses $P_{j}^{i}>0$ for $i=1, \ldots, n$ (respectively, $P_{j}^{C}>0$ ) if there is no consortium (respectively, if the consortium is formed).

- Stage 2: Each library (or the consortium) decides the bundle(s) to buy.

Notice that at stage 1 , we require $P_{j}^{i}>0$, and exclude $P_{j}^{i}=0$, because in some cases publisher $j$ earns a library's entire budget, and thus there is no budget left for publisher $k(\neq j)$. Then our assumption of positive prices rules out the possibility that publisher $k$ gives away bundle $k$ for free. Thus, we suppose that each publisher prefers not selling its bundle to selling it at zero price, which can be justified if there is a small cost of contracting with a library.

Our analysis can be applied to voluntary consortium as long as the participating libraries can commit not to dissolve the consortium at later stages after the consortium formation at stage 0 .

Consider competition in the market for a given library $i(=1, \ldots, n, C)$. We eliminate the superscript $i$ and, without loss of generality, assume $U_{A} \geq U_{B}$. Then, from our previous papers, we have ${ }^{16}$

Lemma 1 (Jeon and Menicucci, 2006 and 2011) Consider competition between two publishers in the market for a given library:

(i) if $M \leq U_{A}-U_{B}$, then publisher $A$ charges $P_{A}=M$, publisher $B$ charges an arbitrary $P_{B}>0$, and the library buys only bundle $A$;

(ii) if $U_{A}-U_{B}<M<U_{A}+U_{B}$, then the publishers charge $P_{A}=\frac{1}{2}\left(M+U_{A}-U_{B}\right)$, $P_{B}=\frac{1}{2}\left(M+U_{B}-U_{A}\right)$, and the library buys both bundles;

(iii) if $U_{A}+U_{B} \leq M$, then the publishers charge $P_{A}=U_{A}, P_{B}=U_{B}$, and the library buys both bundles.

\footnotetext{
${ }^{16}$ In fact, in Jeon and Menicucci $(2006,2011)$, we consider more than two publishers and assume that publishers play a three-stage game in which first each publisher simultaneously decides whether to be active or not, and then only active publishers compete in prices (libraries cannot buy from inactive publishers). In Section 7.2, where we consider three publishers, we adopt this three-stage game. However, when there are only two publishers, this game yields the same outcome that is described by Lemma 1 for our current game.
} 
In particular, when $M \leq U_{A}-U_{B}$, only publisher A succeeds in selling its bundle because even when A charges $P_{A}=M$ (the highest feasible price), the library's payoff from buying only bundle $A, U_{A}$, is larger than the payoff from buying only bundle $B$, $U_{B}+M-P_{B}$, for any $P_{B}>0$. On the other hand, if $M \geq U_{A}-U_{B}$, then the library buys both bundles and it is simple to see that $P_{A}=U_{A}, P_{B}=U_{B}$ when $M \geq U_{A}+U_{B}$. When instead $U_{A}-U_{B}<M<U_{A}+U_{B}$, prices are determined by the indifference condition

$$
U_{A}-P_{A}=U_{B}-P_{B}
$$

and by the binding budget constraint

$$
P_{A}+P_{B}=M
$$

where (1) means that the library is indifferent between purchasing only bundle $A$ and purchasing only bundle $B$. Thus no publisher $j$ has an incentive to increase its price above $P_{j}$ since then the library can not afford to buy both bundles (because of the binding budget constraint) and would buy only the bundle of the rival publisher.

Lemma 1 applies both to each library without a consortium and to the consortium. In the next sections, we compare the outcome without a consortium and the outcome with a consortium.

\section{Consortium of $n$ libraries for exogenous budget}

In this section, we consider the model of $n$ libraries introduced in Section 2. We assume

Assumption 1: $M^{i}<U_{A}^{i}+U_{B}^{i}$ for $i=1, \ldots, n$.

If Assumption 1 is not satisfied for library $i$, there is no competition between the two publishers in the market for library $i$ since each publisher extracts the full surplus. Hence, this assumption implies that the two publishers compete because of the budget constraint in the market for each given library $i=1, \ldots, n$. As a consequence, every library $i$ ends up spending its whole budget to buy one bundle or both. ${ }^{17}$ Assumption 1 also implies that $M^{C}<U_{A}^{C}+U_{B}^{C}$, and thus the consortium spends its whole budget to buy one bundle or both. Therefore, in order to determine the effects of building a consortium on libraries' payoffs, we only need to study how libraries' consumption of bundles is affected.

Without loss of generality, we assume $\Delta^{C} \equiv U_{A}^{C}-U_{B}^{C} \geq 0$ and that there exists an $n^{\prime}$ between 1 and $n$ such that $\Delta^{i} \equiv U_{A}^{i}-U_{B}^{i} \geq 0$ for $i=1, \ldots, n^{\prime}$ and $\Delta^{i}<0$ for $i=n^{\prime}+1, \ldots, n$. Libraries $1, \ldots, n^{\prime}$ are called type A libraries (there is a non-empty set of type A libraries since $U_{A}^{C} \geq U_{B}^{C}$ ); the other libraries (if any) are called type B libraries.

\footnotetext{
${ }^{17}$ In Section 5, in which we make the budget endogenous, Assumption 1 is always satisfied
} 
Lemma 1 makes clear that the only characteristics of library $i$ which matter are $\Delta^{i}$ and $M^{i}$. Without a consortium, library $i$ of type $j$ buys only bundle $j$ if $M^{i} \leq\left|\Delta^{i}\right|$, buys both if $M^{i}>\left|\Delta^{i}\right|$, for $j=A, B$. Likewise, the consortium buys only bundle $A$ if $M^{C} \leq \Delta^{C}$ (recall that $\Delta^{C} \geq 0$ ), buys both if $M^{C}>\Delta^{C}$. These remarks deliver the following results.

Proposition 1 (exogenous budget) Suppose Assumption 1 and (without loss of generality) $\Delta^{C} \equiv U_{A}^{C}-U_{B}^{C} \geq 0$. Consider the consortium of $n(\geq 2)$ libraries.

(i) When $\Delta^{C}<M^{C}$, the consortium buys both bundles and hence the payoff of each library is weakly larger than without the consortium. The consortium strictly increases the aggregate payoff of the libraries unless each library buys both bundles without the consortium.

(ii) When $M^{C} \leq \Delta^{C}$, the consortium buys only bundle $A$ and hence the payoff of each library is weakly smaller than without the consortium. The consortium strictly reduces the aggregate payoff of the libraries unless each library buys only bundle A without the consortium.

Proof. It is omitted since it is straightforward from Lemma 1.

It is simple to see why this proposition is true. Without the consortium, each library of type $j$ either buys only bundle $j$ or both. When the consortium is formed and $M^{C} \leq \Delta^{C}$, each library consumes only bundle $A$ and therefore (i) a type $B$ library is worse off; (ii) a type $A$ library is unaffected if it buys only bundle $A$ without the consortium and is worse off otherwise. On the other hand, when the consortium buys both bundles, each library enjoys maximal consumption and this strictly increases the payoff of each library which does not buy both bundles without the consortium.

Proposition 1 implies that a key issue is whether or not the inequality $\Delta^{C}<M^{C}$ holds. This condition is most easily satisfied when the preferences of libraries over journals are quite heterogenous, that is within the consortium the intensity of the preferences of type A libraries for bundle $A$ over bundle $B$ are more or less counterbalanced by that of type B libraries for bundle $B$ over bundle $A$. The ideal case is such that $\Delta^{1}+\ldots+\Delta^{n^{\prime}}=$ $-\left(\Delta^{n^{\prime}+1}+\ldots+\Delta^{n}\right)$, that is $\Delta^{C}=0$, which makes $\Delta^{C}<M^{C}$ hold for any level of budget of the consortium. For instance, when $n=2$, this occurs if $\Delta^{1}=-\Delta^{2}$. If instead $\Delta^{1}+\ldots+\Delta^{n^{\prime}}$ is much larger than $-\left(\Delta^{n^{\prime}+1}+\ldots+\Delta^{n}\right)$, then $\Delta^{C}$ is much larger than zero and the consortium buys only bundle $A$ if its budget is small. Therefore, the more diverse the libraries are in terms of their preferences for bundles of journals, the more likely forming a consortium is to be beneficial. 


\section{Consortium of two libraries for exogenous budget}

In this section, we analyze our model for the case of two libraries (i.e., $n=2$ ) in order to obtain more precise results by focusing on the correlation between the two libraries' preferences. We maintain Assumption 1 and, to reduce the number of cases to consider, assume $M^{1}=M^{2} \equiv M$.

As in the previous section, we define $\Delta^{i} \equiv U_{A}^{i}-U_{B}^{i}$ for $i=1,2$ and, without loss of generality, assume $\Delta^{1} \geq\left|\Delta^{2}\right| \geq 0$ (with at least one strict inequality). In words, Library 1 prefers bundle $A$ to bundle $B$. If Library 2 also prefers bundle $A$, then Library 1 prefers bundle $A$ more than Library 2 does. If, conversely, Library 2 prefers bundle $B$, then Library 1 prefers bundle $A$ more than Library 2 prefers bundle $B$.

In order to simplify notation, let $\rho \equiv \Delta^{2} / \Delta^{1} \in[-1,1]$ and $\Delta \equiv \Delta^{1}$. Notice that $\rho$ is a measure of the correlation between the two libraries' preferences. With this notation we have

$$
U_{A}^{1}-U_{B}^{1}=\Delta, \quad U_{A}^{2}-U_{B}^{2}=\rho \Delta, \quad U_{A}^{C}-U_{B}^{C}=(1+\rho) \Delta(\geq 0) .
$$

From Lemma 1 and Proposition 1, in the absence of the consortium, Library 1 buys both bundles if and only if $M>\Delta$, Library 2 buys both bundles if and only if $M>|\rho| \Delta$, and the consortium buys both bundles if and only if $M>(1+\rho) \Delta / 2$. Hence, we have:

Observation: If each library individually buys both bundles in the absence of the consortium (i.e., if $M>\Delta$ ), then the consortium buys both bundles, and thus the consortium has no effect.

From now on, we restrict attention to the case of $M \leq \Delta$ and therefore Library 1 buys only bundle $A$ in the absence of the consortium. We can further simplify notation by considering a normalized budget $M^{\prime} \equiv M / \Delta \in(0,1]$. Hence, in what follows, the model has only two parameters: $M^{\prime} \in(0,1]$ and $\rho \in[-1,1]$. From these remarks and Proposition 1, we obtain the next proposition.

Proposition 2 (exogenous budget and correlation) Suppose Assumption 1 and $M^{\prime} \equiv$ $M / \Delta \in(0,1]$. Then Library 1 buys only bundle $A$ in the absence of the consortium. Consider now the consortium of the two libraries.

(i) If $M^{\prime}>(1+\rho) / 2$, the consortium buys both bundles, which strictly increases the libraries' aggregate payoff.

(ii) If $M^{\prime} \leq(1+\rho) / 2$, the consortium buys bundle A only. This reduces the libraries' total payoff if $M^{\prime}>\rho$, but it does not affect any library's consumption nor its payoff if $M^{\prime} \leq \rho$.

Proof. It is omitted since it is straightforward from Lemma 1. 


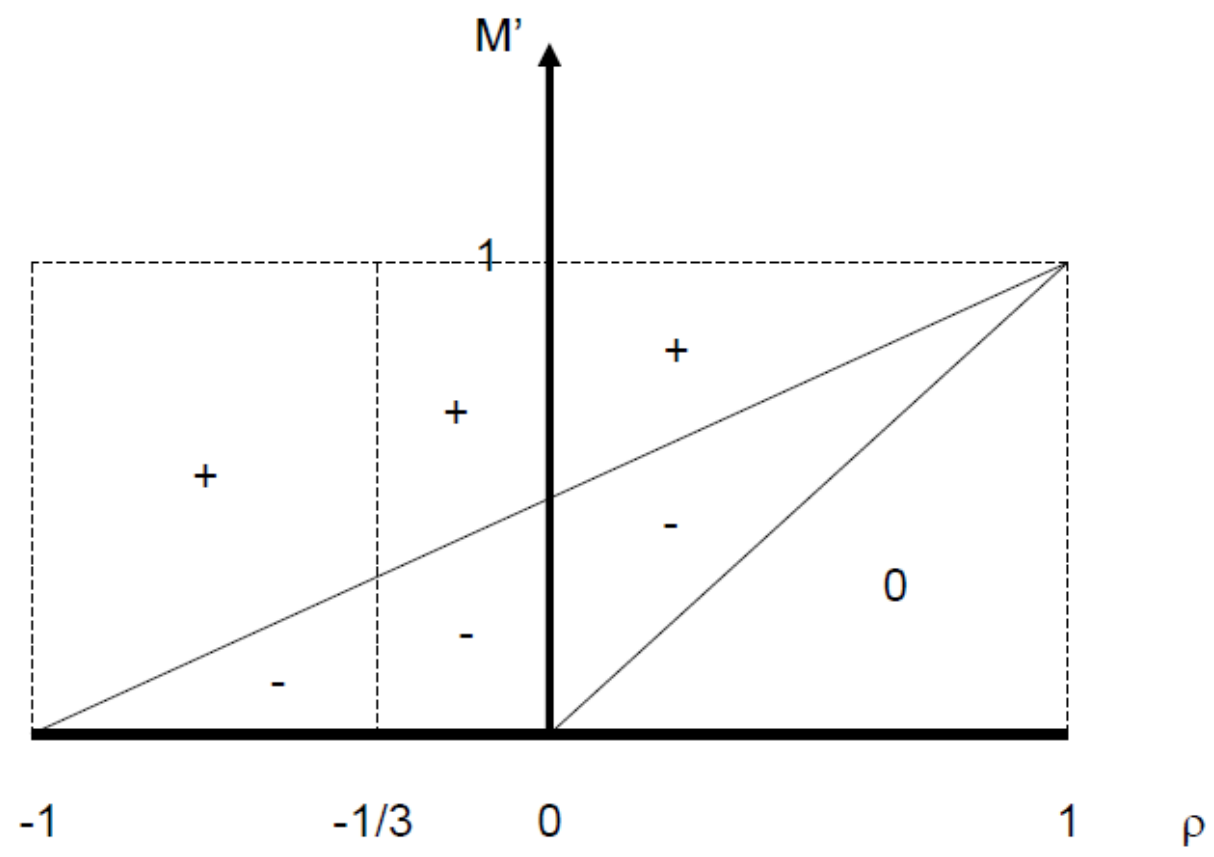

Figure 1: The effect of the consortium under Assumption 1 when the budget is exogenous

Figure 1 represents the sets of $\left(\rho, M^{\prime}\right) \in[-1,1] \times(0,1]$ which satisfy the conditions in Proposition 2(i) and (ii). The region denoted by + is such that $M^{\prime}>(1+\rho) / 2$; the region denoted by - is such that $\rho<M^{\prime} \leq(1+\rho) / 2$; the region denoted by 0 is such that $M^{\prime} \leq \rho<(1+\rho) / 2$. As a corollary, we have:

Corollary 1 Suppose Assumption 1 and $M \leq \Delta$. Consider the consortium of two libraries.

(i) In the case of perfectly negative correlation, the consortium always strictly increases the libraries' total payoff.

(ii) In the case of perfectly positive correlation, the consortium has no impact on the libraries' total payoff.

In order to provide an intuition, let us first consider the extreme case of two identical libraries. Then the consortium has no impact on the payment and the consumption of each library. More generally, Proposition 2(ii) and Figure 1 show that the consortium has no impact as long as the degree of positive correlation is strong enough with respect to the budget, i.e. if $M^{\prime} \leq \rho$. Thus, every library consumes only bundle $A$ regardless of whether or not the consortium is formed. 
Let us now consider the other extreme case of perfectly negative correlation (i.e., $\rho=-1)$. Then, in the absence of the consortium, each library consumes only its preferred bundle: Library 1 consumes only bundle $A$ and Library 2 consumes only bundle $B$. On the contrary, after the consortium is formed, it buys both bundles. This occurs because the opposite preferences of the libraries make the market power of each publisher symmetric under the consortium, and this creates a level-playing field for the two publishers (without affecting the profit of any publisher).

Now let us consider the middle case of no correlation (i.e., $\rho=0$ ). Then, in the absence of the consortium, Library 1 consumes only bundle $A$ and Library 2 consumes both bundles. In this case, the consortium increases (respectively, reduces) the aggregate payoff of the libraries if its budget is large enough, i.e., if $M^{\prime}>1 / 2$ (respectively, small enough, i.e., $1 / 2 \geq M^{\prime}$ ). If the budget is small, publisher $\mathrm{A}$ can export its residual monopoly power from Library 1 to Library 2 in order to monopolize the market of the consortium (and increase its profit). On the contrary, if the budget is large enough, publisher A's market power is not strong enough to monopolize the entire market of the consortium and therefore the consortium buys both bundles (but the profit of publisher $A$ still increases). ${ }^{18}$

Another way to see that a lower $\rho$ makes it more likely that a consortium is beneficial consists in noticing that in order to buy both bundles, the consortium needs to have a budget larger than $(1+\rho) \Delta$. Therefore, if a consortium is formed to buy both bundles, the required budget for the consortium increases with $\rho \in[-1,1]$.

\section{Endogenous budget}

In this section, we relax the assumption of exogenously given budget and continue to analyze the case of two libraries. We consider a single funding agency which allocates funds either to each library separately or to the consortium of the two libraries. For this purpose, we consider a timing in which Stage 0 in Section 2 is modified as follows:

- Stage 0: The funding agency decides whether it will allocate its funds to each library separately or to the consortium and accordingly determines the budget of each library or the budget of the consortium. The consortium is formed only if the funding agency allocates its funds to the consortium.

We assume that the funding agency fully internalizes each library's surplus from journal consumption but does not internalize at all the publishers' payoffs. Therefore, the

\footnotetext{
${ }^{18}$ As the analysis of the three cases $\rho=-1, \rho=0, \rho=1$ suggests, under the consortium the profit of $A$ (the profit of $B$ ) is weakly higher (weakly smaller) than without the consortium, for any $M^{\prime} \leq 1$ and any $\rho \in[-1,1]$.
} 
funding agency's objective is equal to the sum of the libraries' utilities from journal consumption minus the total payments to the publishers. We assume that at Stage 0, the funding agency maximizes this objective.

Consider the market for library $i=1,2, C$, for instance, with $U_{A}^{i} \geq U_{B}^{i}$. According to Lemma 1 , any positive $M^{i}$ smaller than $U_{A}^{i}-U_{B}^{i}$ allows the library to consume bundle $A$ and any $M^{i}$ higher than $U_{A}^{i}-U_{B}^{i}$ and lower than $U_{A}^{i}+U_{B}^{i}$ allows the library to consume both bundles. ${ }^{19}$ The payoff of the funding agency from this market is $U_{A}^{i}-M^{i}$ in the first case and is $U_{A}^{i}+U_{B}^{i}-M^{i}$ in the second case. Since the funding agency wants to minimize the payment to the publishers given the consumption of library $i$, the Supremum of the agency's payoff when $M^{i}$ is endogenous is given by $U_{A}^{i}$ in the first case, and by $2 U_{B}^{i}$ in the second case. Therefore, we have:

Lemma 2 Consider competition between two publishers in the market for a given library when its budget is endogenously chosen by the funding agency. Assume $U_{A} \geq U_{B}$ without loss of generality. Then, the Supremum of the funding agency's payoff is $\max \left\{U_{A}, 2 U_{B}\right\}$.

Proof. It was given in the previous paragraph.

Let $(\mathrm{S}, \mathrm{D}),{ }^{20}$ for instance, represent the equilibrium allocation without the consortium in which Library 1 consumes a single bundle and Library 2 consumes both bundles; (S,S), $(\mathrm{D}, \mathrm{S})$ and $(\mathrm{D}, \mathrm{D})$ are similarly defined. As in the previous section, we use $\Delta \equiv U_{A}^{1}-U_{B}^{1}>0$, $\rho \equiv \frac{U_{A}^{2}-U_{B}^{2}}{\Delta}$, and, without loss of generality, assume $\rho \in[-1,1]$.

In the next lemma, we consider the case of $\rho \geq 0$ (positive correlation). We have:

Lemma 3 (positive correlation) Consider the case of endogenous budget with positive correlation between the two libraries' preferences (i.e., $\rho>0$ ).

(i) In the case of $(S, S)$ or $(D, D)$, building the consortium has no effect on the bundle(s) consumed and on the payoff of the funding agency.

(ii) In the case of $(S, D)$ or $(D, S)$, building the consortium changes the bundle(s) consumed and strictly reduces the payoff of the funding agency.

Proof. It is given in the next paragraphs.

Consider first the case of $(\mathrm{S}, \mathrm{S}): U_{A}^{i} \geq 2 U_{B}^{i}$ holds for $i=1,2$. Therefore, $U_{A}^{C} \geq 2 U_{B}^{C}$ is satisfied. As a consequence, the funding agency induces the consortium to consume only bundle $A$ and thus building a consortium has no effect on the bundle consumed and on

\footnotetext{
${ }^{19}$ As is written in Section 2, we assume that each publisher prefers selling its bundle at any positive price to not selling it. If billing and providing access to the bundle cost $c>0$ to a publisher such that the lowest price at which a publisher wants to sell its bundle is $c$, we can extend our analysis to this case at the cost of having an extra parameter $c$. Our results would hold as long as $c$ is small enough.

${ }^{20} \mathrm{~S}$ refers to "single" and D refers to "duopoly".
} 
the agency's payoff. The same logic applies to the case of (D,D), since then $U_{A}^{i}<2 U_{B}^{i}$ holds for $i=1,2$, which implies $U_{A}^{C}<2 U_{B}^{C}$.

Consider now for instance the case of (S,D). Note first that the funding agency cannot achieve this pattern of consumption through a consortium, since under the consortium both libraries consume either bundle $A$ only or both bundles. Moreover, given that the consortium consumes bundle $A$ (or bundles $A$ and $B$ ), the funding agency can achieve the same consumption pattern without the consortium at the same total payment. This implies that under the consortium the funding agency chooses only between two alternatives $(\mathrm{S}, \mathrm{S})$ and $(\mathrm{D}, \mathrm{D})$, a subset of the alternatives available without the consortium. Since the funding agency finds it optimal to choose $(\mathrm{S}, \mathrm{D})$ in the absence of the consortium, a revealed preference argument implies that $(\mathrm{S}, \mathrm{D})$ gives a higher payoff than $(\mathrm{S}, \mathrm{S})$ or $(\mathrm{D}, \mathrm{D})$. Therefore building a consortium reduces the payoff of the funding agency.

Now we consider the case of $\rho<0$ (negative correlation). In order to reduce the number of cases, we assume that each library obtains the same total utility from consuming both bundles:

Assumption 2: $\left(U_{A}^{1}+U_{B}^{1}\right) / 2=\left(U_{A}^{2}+U_{B}^{2}\right) / 2 \equiv U$.

In the assumption, $U$ represents the average utility from the two bundles. We can normalize each utility $U_{j}^{i}$ by dividing it by $\Delta$ : let $U_{j}^{i \prime}=U_{j}^{i} / \Delta$ for $i=1,2, C$ and $j=A, B$. Let $U^{\prime} \equiv U / \Delta$. Hence, we have:

$$
\begin{aligned}
\left(U_{A}^{1 \prime}, U_{B}^{1 \prime}\right) & =\left(U^{\prime}+1 / 2, U^{\prime}-1 / 2\right), \quad\left(U_{A}^{2 \prime}, U_{B}^{2 \prime}\right)=\left(U^{\prime}-|\rho| / 2, U^{\prime}+|\rho| / 2\right), \\
\left(U_{A}^{C \prime}, U_{B}^{C \prime}\right) & =\left(2 U^{\prime}+(1-|\rho|) / 2,2 U^{\prime}-(1-|\rho|) / 2\right) .
\end{aligned}
$$

$U^{\prime}$ must be larger than $1 / 2$ since $U_{B}^{1 \prime}>0$. Given this normalization, we have only two parameters: $U^{\prime}>1 / 2$ and $\rho \in[-1,0)$. We have:

Lemma 4 (negative correlation) Suppose that Assumption 2 holds, and consider the case of endogenous budget with negative correlation between the two libraries' preferences (i.e., $\rho<0)$.

(i) When $U^{\prime} \geq \frac{3}{2}$ holds. For any $\rho<0,(D, D)$ arises in the absence of the consortium. Under the consortium, the libraries still consume both bundles but at strictly lower prices.

(ii) When $3 / 2>U^{\prime}>1 / 2$ holds, there are two cases:

(a) For $-1 / 3 \leq \rho<0$ : In the absence of the consortium, only $(S, D)$ arises. Under the consortium, the libraries consume both bundles if and only if $U^{\prime} \geq 3(1-|\rho|) / 4$. The consortium strictly increases the payoff of the funding agency if and only if $U^{\prime}>(3-4|\rho|) / 2$.

(b) For $-1 \leq \rho<-1 / 3$ : In the absence of the consortium, $(S, D)$ arises if $U^{\prime}>3|\rho| / 2$ and $(S, S)$ arises otherwise. Under the consortium, the libraries always 
consume both bundles. The consortium strictly increases the payoff of the funding agency if and only if $U^{\prime}>\frac{1}{2} \max \left\{3-4|\rho|, \frac{3-|\rho|}{2}\right\}$.

Proof. It is straightforward and hence is omitted.

This lemma reveals first that, for any $\rho<0$, whenever the average value of the journals is large enough (i.e., $U^{\prime} \geq 3 / 2$ ) such that $(\mathrm{D}, \mathrm{D})$ arises without a consortium, then the consortium strictly increases the payoff of the funding agency. This is because building the consortium does not affect consumption but reduces the gap between the willingness to pay for bundle $A$ and the one for bundle $B$; this in turn increases competition between the two publishers and allows the libraries to consume both bundles at a lower total price. Precisely, without the consortium the total price paid is $1+|\rho|$ but the consortium pays only $1-|\rho|$.

When the average value of the bundles is not large (i.e., $3 / 2>U^{\prime}>1 / 2$ ), either $(\mathrm{S}, \mathrm{D})$ or $(\mathrm{S}, \mathrm{S})$ occurs without a consortium. To sharpen the intuition, let us consider the two extreme cases of perfect negative correlation and no correlation. Under perfect negative correlation, building a consortium always strictly increases the payoff of the funding agency, which is the same as the aggregate payoff of the libraries. In this case, only $(\mathrm{S}, \mathrm{S})$ arises in the absence of the consortium: Library 1 consumes only bundle $A$ and Library 2 consumes only bundle $B$. Then, building a consortium creates a level playing field between the two publishers such that the consortium can consume both bundles at almost zero price. In contrast, in the extreme case of no correlation, only (S,D) occurs without a consortium. Then, because of the revealed preference argument explained right after Lemma 3, building a consortium always strictly reduces the payoff of the funding agency. For the general case of negative correlation (i.e., $0>\rho>-1$ ), there exists a cutoff value of $U^{\prime} \equiv U / \Delta$ such that building a consortium strictly increases the payoff of the funding agency for any $U^{\prime}$ above the threshold. This cut-off strictly decreases with the absolute degree of the negative correlation $|\rho|$.

Figure 2 describes the consumption patterns in the absence of the consortium under Assumption 2. Figure 3 shows the region (marked with + ) in which building the consortium strictly increases the payoff of the funding agency, the region (marked with 0) in which building the consortium does not affect it, and the region (marked with -) in which building the consortium strictly reduces it. Summarizing, we have:

Proposition 3 (endogenous budget and correlation) Consider the case of endogenous budget.

(i) When the two libraries' preferences are positively correlated (i.e., $\rho \geq 0$ ), building a consortium either has no effect on the payoff of the funding agency, or strictly reduces it. Under Assumption 2, the range of $U^{\prime} \equiv U / \Delta$ for which the consortium is harmful shrinks with the degree of correlation such that it disappears for perfect positive correlation. 


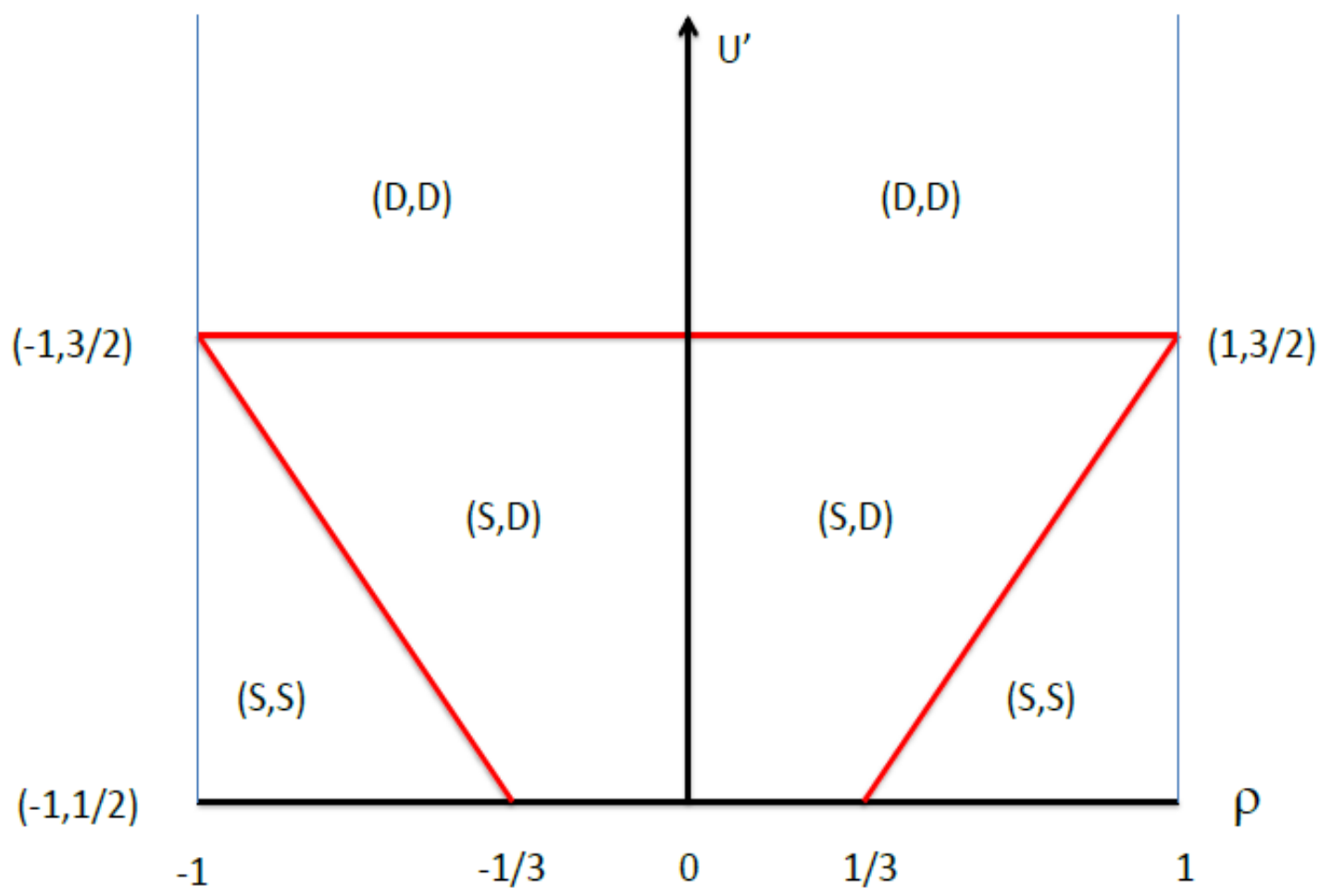

Figure 2: The consumption patterns without a consortium under Assumption 2 when the budget is endogenous 


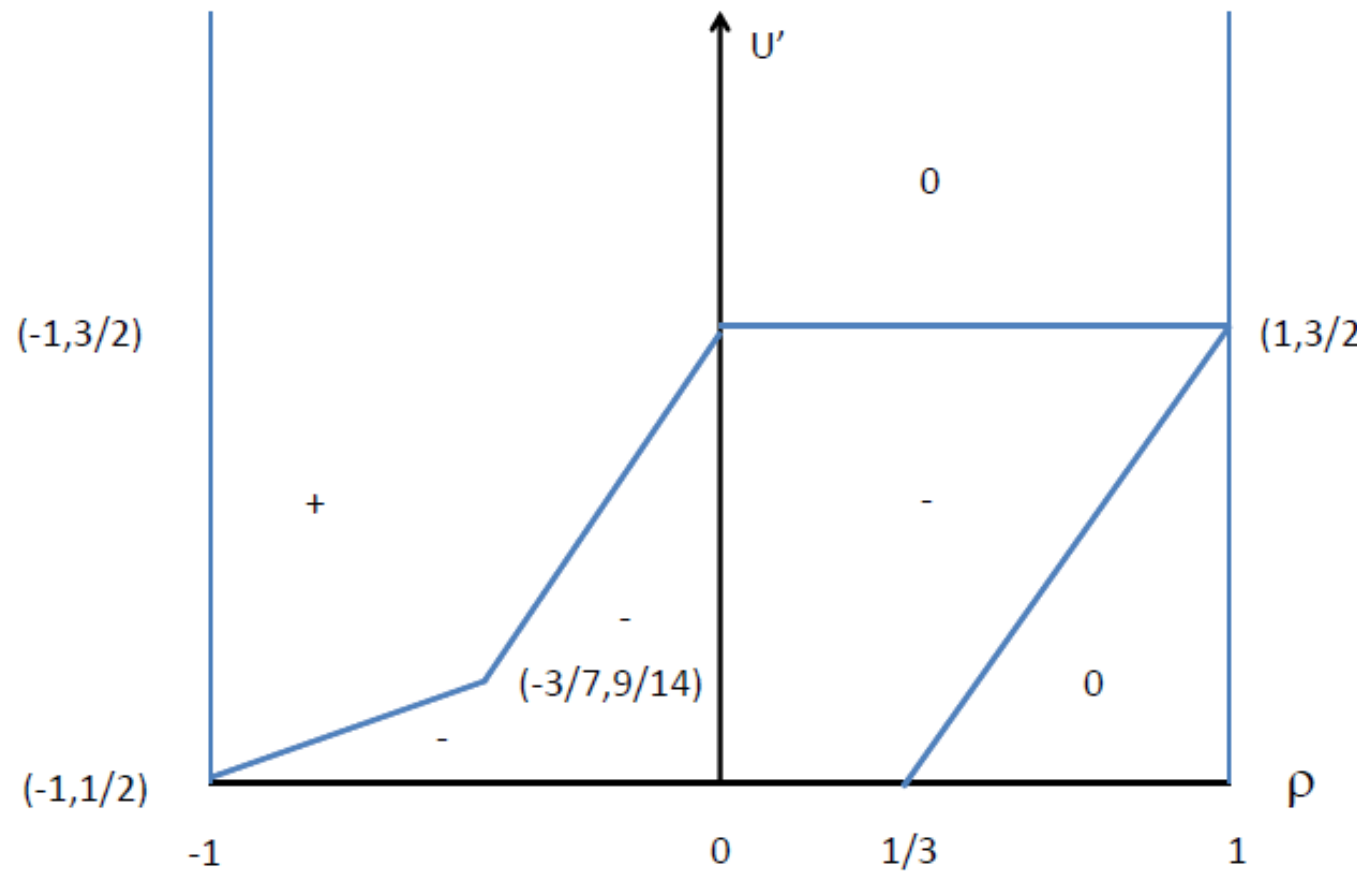

Figure 3: The effect of the consortium under Assumption 2 when the budget is endogenous

(ii) When the two libraries' preferences are negatively correlated (i.e., $\rho<0$ ), under Assumption 2, there exists a cut-off value of $U^{\prime}$ such that building a consortium strictly increases the payoff of the funding agency for any $U^{\prime}$ above the threshold. This cut-off strictly decreases with the absolute degree of negative correlation $|\rho|$ such that the consortium always increases the payoff of the funding agency for perfect negative correlation.

Proof. It is straightforward and hence is omitted.

Corollary 2 Consider the case of endogenous budget and suppose Assumption 2.

(i) In the case of perfectly negative correlation, building the consortium always strictly increases the payoff of the funding agency.

(ii) In the case of perfectly positive correlation, building the consortium has no impact on the payoff of the funding agency.

When we compare Figure 1 of exogenous budget and Figure 3 of endogenous budget, it is remarkable that they share a number of features even if the parameter represented on the vertical axis is different in the two figures. First, Corollary 1 and Corollary 2 have the identical predictions for the two extreme cases of perfect positive and perfect 
negative correlation. Second, given negative correlation, the parameter range for which consortium is beneficial increases with the absolute degree of correlation. Third, given positive correlation, the parameter range for which consortium is harmful decreases with the degree of correlation. The key difference arises for the case of positive correlation: while building a consortium is strictly beneficial for a certain range of parameters when the budget is exogenously given, it can never be strictly beneficial in the case of endogenous budget.

\section{Commitment to consortium pricing and consor- tium trap}

In this section, we consider the case in which each publisher can commit to a price that it will charge to a consortium before the funding agency decides whether or not to form it. We consider two different scenarios. In the first scenario, we allow the publishers to commit only to the prices for the consortium, whereas in the second scenario, we allow them to offer a menu of prices (i.e., prices for individual libraries and prices for the consortium).

We find that building a consortium is always beneficial in the first scenario. In contrast, the outcome of the menu pricing is equivalent to that of the pricing game played after the consortium is formed. In addition, we study a variation of the menu pricing game in which the consortium cannot be formed with a high probability due to transaction costs and find that the outcome of this game is equivalent to that of the first scenario.

We consider the model of exogenous budget in Section 4. There are two libraries, each with an exogenous budget of $M^{\prime} \equiv M / \Delta>0$ where $\Delta \equiv \Delta^{1}$. Recall that $\rho \equiv \Delta^{2} / \Delta^{1} \in$ $[-1,1]$ and $U_{j}^{i \prime} \equiv U_{j}^{i} / \Delta$. We maintain Assumption 1 .

\subsection{Commitment to consortium pricing}

In this subsection, we study the game with the following timing:

- Stage 0: Each publisher $j(=A, B)$ simultaneously proposes a price $P_{j}^{C}$ it will charge if the consortium is formed.

- Stage 1: The funding agency decides whether to form a consortium or not.

- Stage 2: If the consortium is formed, it makes purchase decisions given $\left(P_{A}^{C}, P_{B}^{C}\right)$. Otherwise, each publisher $j(=A, B)$ simultaneously proposes a price $P_{j}^{i}$ to each library $i(=1,2)$ and then each library makes purchase decisions. 
Under the above timing, if the consortium is not formed, each library obtains the payoff associated with the outcome described in Lemma 1, which becomes its reservation utility. Let $\pi_{j}^{i *}$ be the equilibrium profit that publisher $j$ obtains from library $i$ in the absence of the consortium. We prove that no publisher realizes a profit strictly higher than the one without the consortium. For instance, suppose that publisher $A$ chooses $P_{A}^{C}>\pi_{A}^{1 *}+\pi_{A}^{2 *}$, that is $A$ charges a price strictly higher than its total profit without the consortium. For bundle $A$ to be sold to the consortium, the libraries' joint payoff under the consortium should be higher than the one without it; that is, the aggregate participation constraint (written with respect to the sum of the reservation utilities) must be satisfied. However, $P_{A}^{C}>\pi_{A}^{1 *}+\pi_{A}^{2 *}$, together with the consortium budget constraint, implies that if the consortium buys bundle $A$, then $B$ 's profit is at most $2 M^{\prime}-P_{A}^{C}$, which is strictly lower than $B$ 's profit without the consortium. In the proof of Lemma 5 , we prove that by choosing $P_{B}^{C}$ very high, $P_{B}^{C}=\infty$ for instance, $B$ can veto the consortium formation since $P_{A}^{C}\left(>\pi_{A}^{1 *}+\pi_{A}^{2 *}\right)$ and $P_{B}^{C}=\infty$ together violate the aggregate participation constraint. In this way, $B$ realizes a profit equal to $\pi_{B}^{1 *}+\pi_{B}^{2 *}$, which is strictly higher than $2 M^{\prime}-P_{A}^{C}$. We have:

Lemma 5 Consider the game in which each publisher can commit to a price that it will charge to the consortium before the funding agency decides to form it. Under Assumption 1, no publisher $j(=A, B)$ can realize a profit strictly higher than its profit without the consortium, $\pi_{j}^{1 *}+\pi_{j}^{2 *}$.

Proof. See Appendix A.

Lemma 5 suggests that there is an equilibrium in which each publisher $j$ commits to $P_{j}^{C}=\pi_{j}^{1 *}+\pi_{j}^{2 *}$ and the consortium is always formed. In this equilibrium, the libraries never lose from building a consortium.

Proposition 4 Consider the game in which each publisher can commit to a price that it will charge to the consortium before it is formed by a funding agency. Under Assumption 1 , there is an equilibrium in which each publisher $j$ commits to $P_{j}^{C}=\pi_{j}^{1 *}+\pi_{j}^{2 *}$. In this equilibrium,

(i) if $M^{\prime}>1$, the funding agency is indifferent between forming a consortium and not forming it: each library consumes both bundles regardless of the consortium formation.

(ii) if $M^{\prime} \leq \rho$ (with $\rho>0$ ), the funding agency is indifferent between forming a consortium and not forming it: each library consumes only bundle A regardless of the consortium formation.

(iii) if $1 \geq M^{\prime}>\max \{\rho, 0\}$, the funding agency strictly gains from forming a consortium. Without the consortium, Library 1 consumes only bundle A and Library 2 consumes either both bundles or only bundle B. After building the consortium, each library consumes both bundles. 
Proof. We just prove the existence of the equilibrium as the rest is straightforward. The proof of Lemma 5 shows that given $P_{j}^{C}=\pi_{j}^{1 *}+\pi_{j}^{2 *}$, if publisher $k(\neq j)$ deviates from $P_{k}^{C}=\pi_{k}^{1 *}+\pi_{k}^{2 *}$ by increasing its price, then the libraries cannot afford to buy both bundles through the consortium, and rather than buying only bundle $k$, they prefer not to form the consortium. Therefore, there is no profitable deviation for publisher $k$.

The commitment to consortium pricing may correspond to a short-term situation. In the short run, the publishers can commit to an aggregate payoff at least as large as the payoff that the funding agency obtains under the status quo of no consortium in order to induce it to form a consortium. Actually, in the equilibrium described in Proposition 4, the participation constraint is satisfied not only at the aggregate level but also at the individual level: each library at least weakly obtains a higher payoff under the consortium than without the consortium. This suggests that Proposition 4 holds for the voluntary consortium as well. However, in the long run, the publishers can charge prices which are not constrained any more by the aggregate participation constraint as is shown in the next subsection. Note also that it is the largest publisher (i.e., publisher $A$ ) which gains from the consortium.

\subsection{Menu pricing}

In this subsection, we allow each publisher to offer a menu of prices (i.e., prices for individual libraries and prices for the consortium) and study the game with the following timing:

- Stage 0: Each publisher $j(=A, B)$ simultaneously proposes a menu of prices $\left\{P_{j}^{1}, P_{j}^{2}, P_{j}^{C}\right\}$ which it will charge depending on whether a consortium is formed.

- Stage 1: The funding agency decides whether to form a consortium or not.

- Stage 2: The libraries or the consortium makes purchase decisions.

Let $\pi_{j}^{C *}$ be the equilibrium profit that publisher $j$ obtains when the publishers compete after the consortium is formed. The next proposition describes the unique equilibrium outcome of the game with the above timing:

Proposition 5 Consider the game in which each publisher can commit to a menu of prices for individual libraries and for the consortium before the funding agency decides whether or not to form a consortium. Under Assumption 1, the unique equilibrium outcome of this game is the same as that of the game in which the publishers offer prices after the consortium is formed. 


\section{Proof. See Appendix A.}

The reason why the menu pricing game leads to the outcome of the pricing game played after the consortium is formed is the following. Given that the funding agency maximizes the aggregate payoff of the libraries and can form the consortium for this purpose, what matters is $U_{j}^{C}$ in terms of its willingness to pay and $M^{C}\left(=2 M^{\prime}\right)$ in terms of its ability to pay. Then, regardless of whether publisher $k(\neq j)$ sells its bundle through the individual libraries or the consortium, the total surplus to the libraries from bundle $k$ is $U_{k}^{C}$. Therefore, whenever publisher $k$ tries to earn strictly more than $\pi_{k}^{C *}$, publisher $j$ can offer $P_{j}^{1}=P_{j}^{2}=\infty$ and $P_{j}^{C}=\pi_{j}^{C *}$. Then, the funding agency must choose between buying only bundle $j$ and buying only bundle $k$, and prefers buying only bundle $j$ at the price of $\pi_{j}^{C *}$.

Finally, we study the following variation of the menu pricing game. After the publishers offer their prices, a transaction cost for building the consortium is realized. It is zero with probability $\lambda>0$ and it is prohibitively high (and hence the consortium cannot be formed) with probability $1-\lambda$. The distribution of the transaction cost is common knowledge. When $\lambda$ is close to zero, we have:

Proposition 6 Suppose Assumption 1. The outcome of the game with a commitment to consortium pricing described in Proposition 4 is the same as that of the menu pricing game if the probability of having a very high transaction cost is high enough: both the consortium and each library face the same prices in both games.

\section{Proof. See Appendix A.}

Proposition 6 provides an additional justification to the outcome of Proposition 4. When the probability of failing to build the consortium is high enough, no publisher can threaten to shut down the trade through individual libraries and therefore the option to trade through individual libraries determines a reservation utility for each library.

However, once the consortium is formed, it reveals that the transaction cost is not high. Then, a publisher can threaten to charge a very high price to each library when the consortium is not formed. Therefore, publishers' pricing may not be constrained any more by the reservation utility generated from the ability to purchase journals individually. Then, those libraries with $1 \geq M^{\prime}>(1+\rho) / 2$ will continue to enjoy the benefit of consortium building whereas those with $(1+\rho) / 2 \geq M^{\prime}>\rho$ will experience a loss from consortium building. In the latter case, the libraries are in a "consortium trap".

\section{Extensions}

In this section, in order to show the robustness of our main insight, we briefly describe the results from two extensions of the baseline model of exogenous budget: the detailed 
analysis of each extension is provided in Appendix B of Supporting Information.

\subsection{Complements or substitutes}

We assumed that the utility that a library obtains from the bundle of a publisher does not depend on whether the library has access to the bundle of the other publisher. We can relax this assumption and allow for the possibility that the two bundles are substitutes or complements. More precisely, if library $i$ (with $i=1, \ldots, n$ ) buys both bundles, it gets a gross utility of $U_{A}^{i}+U_{B}^{i}+\delta^{i}$, where $\delta^{i}>0$ represents the case of complements and $\delta^{i}<0$ represents the case of substitutes. We assume that the gross utility from consuming both bundles is greater than the gross utility from a single bundle: $U_{A}^{i}+U_{B}^{i}+\delta^{i}>\max \left\{U_{A}^{i}, U_{B}^{i}\right\}$. We modify Assumption 1 such that the budget constraint binds for any $\delta^{i}$ and that the substitution is not strong enough such that library $i$ prefers buying both bundles to buying a single one when $M^{i}>\left|\Delta^{i}\right|$. Then, we can show that our results regarding when a consortium increases or reduces the aggregate payoff of the libraries (i.e., Proposition 1) does not depend on whether the journals are complements or substitutes. This is mainly because the equilibrium pricing in the market of library $i$ (with $i=1, \ldots, n, C$ ) does not depend on $\left(\delta^{1}, \ldots, \delta^{n}\right)$. For instance, when $M^{i}>\left|\Delta^{i}\right|$, the equilibrium prices of the duopoly are determined by the binding budget constraint $P_{A}^{i}+P_{B}^{i}=M^{i}$ and the indifference condition $U_{A}^{i}-P_{A}^{i}=U_{B}^{i}-P_{B}^{i}$, which do not depend on $\delta^{i}$.

\subsection{Three publishers}

We can also extend our analysis to the case of three publishers. Suppose that there are two libraries. Let $U_{(r)}^{i}$ represent the surplus that library $i(=1,2, C)$ obtains from its $r$-th best $(r=1,2,3)$ bundle: the ranking is specific to $i$. In this case, Assumption 1 can be extended in a straightforward way. We can show that building a consortium reduces (respectively, increases) the aggregate payoff of the libraries if their preferences are similar (respectively, diverse) enough. Namely, when the consortium strongly prefers one bundle over the other two bundles such that $M^{C} \leq U_{(1)}^{C}-U_{(2)}^{C}$ holds, each library is strictly worse off under the consortium, unless each library buys only the bundle preferred by the consortium in its absence. By contrast, if the consortium has sufficiently balanced preferences such that its utility from the two best bundles is not much larger than twice the utility from the worst bundle (i.e., $M^{C}>\left[U_{(1)}^{C}-U_{(3)}^{C}\right]+\left[U_{(2)}^{C}-U_{(3)}^{C}\right]$ holds), each library is strictly better off under the consortium, unless each library buys all three bundles without the consortium. 


\section{Conclusion}

Our findings show that what determines whether libraries gain or lose from forming a consortium is not the mere size of the consortium but its composition, as is emphasized by Dana (2012) in his theory of buyer groups. We find that libraries with similar preferences are likely to lose from building a consortium whereas libraries with diverse preferences are likely to gain from building a consortium. We also find a potential tension between a short-term strategy and a long-term strategy as long as the former dictates forming a library consortium among libraries with similar preferences to benefit from quantity discounts. This tension could lead to a consortium trap in that a short-term gain is associated with a long-terms loss.

As our approach does not take into account the effect that a consortium has on the bargaining power and this force can make it desirable to build a consortium among libraries with similar preferences, we need empirical studies about long-term effects of consortia in order to identify the major forces determining the success or failure of a consortium. ${ }^{21}$

\section{References}

[1] Armstrong, Mark (2010). "Collection Sales: Good or Bad for Journals", Economic Inquiry, 48 (1): 163-176.

[2] Armstrong, Mark (2014). "Opening Access to Research: Theory and Policy", Mimeo.

[3] Autorité de Concurrence, (2007). Décision nº 07-D-09 du 14 mars 2007 relative à des pratiques mises en œuvre par le laboratoire GlaxoSmithKline France.

[4] Bergstrom, Theodore C. (2001). "Free Labor for Costly Journals", Journal of Economic Perspectives, 15: 183-198

[5] Bernheim, Douglas and Michael Whinston (1986). "Menu Auctions, Resource Allocation and Economic Influence." Quarterly Journal of Economics, 101, 1-31.

[6] Bernheim, Douglas and Michael Whinston (1990). "Multi-market Contact and Collusive Behavior." The RAND Journal of Economics, 21(1), 1-26.

[7] Bernheim, Douglas and Michael Whinston (1998). "Exclusive Dealing." Journal of Political Economy, 106, 64-103.

\footnotetext{
${ }^{21} \mathrm{~A}$ (weak) empirical support for the consortium trap is provided by Crawford (2014) who finds that for the period of 2000-2012, U.S. academic libraries' spending on serials started to pick up more rapidly since 2002 after flattening for 2000-2002. He argues that the big deal brought "short-term win and long-term problem" as it did not bring serial price escalation to a manageable level.
} 
[8] Cestone, G . and Fumagalli, C. (2005). "The Strategic Impact of Resource Flexibility in Business Groups." RAND Journal of Economics, 36: 193-214.

[9] Chen, Yuxin and Xinxin Li (2013). "Group Buying Commitment and Sellers' Competitive Advantages", Journal of Economics and Management Strategy, 22:164-183.

[10] Crawford, Walter (2014). Big-deal Serial Purchasing: Tracking the Damage, ALA TechSource, Chicago

[11] Dana, J. (2012). "Buyer Group as Strategic Commitments", Games and Economic Behavior, 74(2): 470-485.

[12] Davis, Philip M. (2002) "Patterns in Electronic Journal Usage: Challenging the Composition of Geographic Consortia." College \& Research Libraries, November, 484-497

[13] Dewatripont M., V. Ginsburgh, P. Legros, A. Walckiers, J.-P. Devroey, M. Dujardin, F. Vandooren, P. Dubois, J. Foncel, M. Ivaldi, M.-D. Heusse (2006). Study on the Economic and Technical Evolution of the Scientific Publication Markets in Europe.

[14] Edlin, Aaron S. and Daniel L. Rubinfeld (2004). "Exclusion or Efficient Pricing: The "Big Deal" Bundling of Academic Journals." Antitrust Law Journal, 72, 119-157

[15] European Commission (1999). Buyer power and its impact on competition in the food retail distribution sector of the European Union: Final report.

[16] Fluck, Z. and Lynch, A. (1999). "Why Do Firms Merge and Then Divest? A Theory of Financial Synergy." Journal of Business, 72: 319-346.

[17] Gatten, Jeffrey N. and Tom Sanville (2004). "An Orderly Retreat from the Big Deal: Is It Possible for Consortia", D-Lib Magazine, 10 (10).

[18] Gertner, R .H., Scharfstein, D.S., and Stein, J.C. (1994). "Internal Versus External Capital Markets." Quarterly Journal of Economics, 109: 1211-1230.

[19] Haank, Derk (2001). "Is electronic publishing being used in the best interests of science? The Publisher's view". Proceedings of the Second ICSU-UNESCO International Conference on Electronic Publishing in Science

[20] Inderst, R and G. Shaffer (2007). "Retail Mergers, Buyer Power and Product Variety", The Economic Journal, 117(516): 45-67. 
[21] Jeon, Doh-Shin and Domenico Menicucci (2006). "Bundling Electronic Journals and Competition among Publishers", Journal of the European Economic Association, September 4(5): 1038-83.

[22] Jeon, Doh-Shin and Domenico Menicucci (2011). "Interconnection among Academic Journal Websites: Multilateral versus Bilateral Interconnection", The RAND Journal of Economics, 42(2): 363-386.

[23] Jeon, Doh-Shin and Domenico Menicucci (2012). "Bundling and Competition for Slots", American Economic Review, 102: 1957-1985.

[24] Jeon, Doh-Shin and Domenico Menicucci (2014). "A Theory of Buyer Fragmentation: Divide-and-conquer Intensifies Competition", TSE Working Paper.

[25] Jeon, Doh-Shin and Jean-Charles Rochet (2010). "The Pricing of Academic Journals: A Two-Sided Market Perspective", American Economic Journal: Microeconomics, 2 , $222-255$.

[26] Marvel, H.P. and H. Yang, (2008). "Group Purchasing, Nonlinear Tariffs, and Oligopoly," International Journal of Industrial Organization, 26(5): 1090-1105.

[27] McCabe, Mark (2004). "Information Goods and Endogenous Pricing Strategies: the Case of Academic Journals." Economics Bulletin, 12 (10)

[28] McCabe, Mark J. and Christopher Snyder, (2007). "Academic Journal Prices in a Digital Age: A Two-Sided Market Approach", The B.E. Journal of Economic Analysis $\&$ Policy, 7(1) (Contributions), Article 2.

[29] Okerson, Ann (2015). "Case Study 5: Embracing Wide Deals," in Library Consortia: Models for Collaboration and Sustainability, eds by Valerie Horton and Greg Pronevitz, ALA Editions, Chicago

[30] Peters, Thomas A. (2001a). "Agile Innovation Clubs", The Journal of Academic Librarianship. 27 (2): 149-151

[31] Peters, Thomas A. (2001b). "What's the big deal?", The Journal of Academic Librarianship. 27 (4): 302-304

[32] Scharfstein, D. and Stein, J.C. (2000). "The Dark Side of Internal Capital Markets: Divisional Rent-Seeking and Inefficient Investment", Journal of Finance, 55: 25372564 . 
[33] Scigliano, Marisa (2010). "Measuring the Use of Networked Electronic Journals in an Academic Library Consortium: Moving beyond MINES for Libraries in Ontario Scholars Portal", Serials Review, 36 (2), 72-78.

[34] Sloan, Bernie (2000). "Understanding Consortia Better: What Vendors Can Learn", Library Journal, March 15: 57-58

[35] Stein, J.C. (1997). "Internal Capital Markets and the Competition for Corporate Resources", Journal of Finance, 52: 111-133.

\section{Appendix A}

\subsection{Proof of Lemma 5}

(i) Consider $M^{\prime}>1$. Hence, each library consumes both bundles without a consortium. If $P_{A}^{C}$ is strictly higher than $\pi_{A}^{1 *}+\pi_{A}^{2 *}$ and $B$ chooses $P_{B}^{C}=\infty$, then the aggregate participation constraint reduces to

$$
U_{A}^{1 \prime}+U_{B}^{1 \prime}+U_{A}^{2 \prime}+U_{B}^{2 \prime} \leq U_{A}^{1 \prime}+U_{A}^{2 \prime}+2 M^{\prime}-P_{A}^{C}
$$

which implies, from the binding budget constraint $2 M^{\prime}=\pi_{A}^{1 *}+\pi_{A}^{2 *}+\pi_{B}^{1 *}+\pi_{B}^{2 *}$,

$$
U_{B}^{1 \prime}+U_{B}^{2 \prime}<\pi_{B}^{1 *}+\pi_{B}^{2 *}
$$

This can never be satisfied since it means that without a consortium, the libraries pay to $B$ more than the surplus they obtain from bundle $B$. The same kind of proof can be applied to show that $B$ cannot earn more than $\pi_{B}^{1 *}+\pi_{B}^{2 *}$.

(ii) Consider $M^{\prime} \leq \rho$ (with $\rho>0$ ). Then it is clear that $A$ cannot earn more than $\pi_{A}^{1 *}+\pi_{A}^{2 *}$ since $\pi_{A}^{1 *}+\pi_{A}^{2 *}=2 M^{\prime}$. Publisher $B$ cannot increase its profit above $\pi_{B}^{1 *}+\pi_{B}^{2 *}=0$ because $A$ can induce the consortium to buy bundle $A$ with $P_{A}^{C}=2 M^{\prime}$ as $2 M^{\prime} \leq U_{A}^{C^{\prime}-}$ $U_{B}^{C^{\prime}}=1+\rho$ holds.

(iii) Consider $1 \geq M^{\prime}>\max \{\rho, 0\}$. In the absence of a consortium, Library 1 consumes only bundle $A$ and Library 2 consumes only bundle $B$ (if $M^{\prime} \leq-\rho$ with $\rho<0$ ) or both bundles (if $M^{\prime}>|\rho|$ ). Consider the case in which Library 2 consumes only bundle $B: M^{\prime} \leq-\rho$ with $\rho<0$. If $P_{A}^{C}$ is greater than $\pi_{A}^{1 *}+\pi_{A}^{2 *}=M^{\prime}$ and $B$ chooses $P_{B}^{C}=\infty$, then the aggregate participation constraint becomes

$$
U_{A}^{1 \prime}+U_{B}^{2 \prime} \leq U_{A}^{1 \prime}+U_{A}^{2 \prime}+2 M^{\prime}-P_{A}^{C},
$$

which implies

$$
U_{B}^{2 \prime}-U_{A}^{2 \prime}<M^{\prime}
$$


The latter inequality is equivalent to $-\rho<M^{\prime}$, which cannot hold as we assume $M^{\prime} \leq-\rho$. The same kind of proof can be applied to show that $B$ cannot earn more than $\pi_{B}^{1 *}+\pi_{B}^{2 *}$.

Consider now the case in which Library 2 consumes both bundles: $M^{\prime} \geq|\rho|$. If $P_{A}^{C}$ is greater than $\pi_{A}^{1 *}+\pi_{A}^{2 *}$ and $B$ chooses $P_{B}^{C}=\infty$, then the aggregate participation constraint becomes

$$
U_{A}^{1 \prime}+U_{A}^{2 \prime}+U_{B}^{2 \prime} \leq U_{A}^{1 \prime}+U_{A}^{2 \prime}+2 M^{\prime}-P_{A}^{C},
$$

which implies

$$
U_{B}^{2 \prime}<\pi_{B}^{2 *}
$$

which can never be satisfied. The same kind of proof can be applied to show that $B$ cannot earn more than $\pi_{B}^{1 *}+\pi_{B}^{2 *}$.

\subsection{Proof of Proposition 5}

We start by proving two lemmas:

Lemma 6 Suppose Assumption 1. Under menu pricing, in any equilibrium the profit of each publisher $j$ is not smaller than $\pi_{j}^{C *}$.

Proof. If $M^{\prime} \leq(1+\rho) / 2$, then $\pi_{A}^{C *}=2 M^{\prime}$ and $\pi_{B}^{C *}=0$. In this case, the result is obvious for publisher $B$. Publisher $A$ can induce the funding agency to form a consortium and buy bundle $A$ by playing $P_{A}^{C}=2 M^{\prime}$ and $p_{A}^{1}=p_{A}^{2}=\infty$.

If $M^{\prime}>(1+\rho) / 2$, then we show that no equilibrium exists such that $\pi_{A}<\pi_{A}^{C *}$. We distinguish the case in which the consortium is formed from the opposite case. Suppose that the consortium is formed and that an equilibrium with $\pi_{A}<\pi_{A}^{C *}$ exists. Then, we must have $\pi_{B}=2 M^{\prime}-\pi_{A}>\pi_{B}^{C *} \cdot{ }^{22}$ Consider the deviation of publisher $A$ which consists in setting $P_{A}^{C}$ slightly higher than $\pi_{A}$ (but smaller than $\pi_{A}^{C *}$ ) and $p_{A}^{1}=p_{A}^{2}=\infty$. After the deviation, if the consortium is formed, it will buy bundle $A$ since $U_{A}^{C}-\pi_{A}^{C *}=U_{B}^{C}-\pi_{B}^{C *}$ implies $U_{A}^{C}-P_{A}^{C}>U_{B}^{C}-\pi_{B}$. But it may occur that the consortium is not formed after the deviation because each individual library buys bundle $B$, obtaining the total payoff of $U_{B}^{C}+2 M^{\prime}-p_{B}^{1}-p_{B}^{2}$. This requires $U_{A}^{C}-\pi_{A} \leq U_{B}^{C}-p_{B}^{1}-p_{B}^{2}$, because if $U_{A}^{C}-\pi_{A}>U_{B}^{C}-p_{B}^{1}-p_{B}^{2}$, then the consortium is formed, given $P_{A}^{C}$ slightly higher than $\pi_{A}$. The inequality $U_{A}^{C}-\pi_{A} \leq U_{B}^{C}-p_{B}^{1}-p_{B}^{2}$ implies $p_{B}^{1}+p_{B}^{2}<\pi_{B}$. Then consider the following deviation of A: $P_{A}^{C}=\infty, p_{A}^{1}+p_{A}^{2}=\pi_{A}+\varepsilon\left(\leq 2 M^{\prime}-p_{B}^{1}-p_{B}^{2}\right)$. If the consortium is formed, then it obtains a payoff of $U_{B}^{C}-\pi_{B}+2 M^{\prime}$, whereas if it is not formed, then the libraries obtain payoff $U_{A}^{C}-\pi_{A}-\varepsilon+U_{B}^{C}-p_{B}^{1}-p_{B}^{2}+2 M^{\prime}$ by purchasing both bundles. The

\footnotetext{
${ }^{22}$ If $\pi_{A}<\pi_{A}^{C *}$ and $\pi_{B}=2 M^{\prime}-\pi_{A}=\pi_{B}^{C *}$, then $\pi_{A}+\pi_{B}<2 M^{\prime}$. In this case, $\mathrm{A}$ has an incentive to raise $\pi_{A}$ at least by $\epsilon>0$ small enough.
} 
latter payoff is greater than $U_{B}^{C}-\pi_{B}+2 M^{\prime}$ because $U_{A}^{C}-\pi_{A}-\varepsilon>0$ and $p_{B}^{1}+p_{B}^{2}<\pi_{B}$. Hence $A$ can increase its payoff above $\pi_{A}$.

Now suppose that there exists an equilibrium in which the consortium is not formed, $\pi_{A}<\pi_{A}^{C *}$ and $\pi_{B}=2 M^{\prime}-\pi_{A}>\pi_{B}^{C *}$. Then consider the deviation of publisher $A$ such that $p_{A}^{1}=p_{A}^{2}=\infty$ and $P_{A}^{C}=\pi_{A}^{C *}$. If the consortium is not formed, the libraries can buy only bundle $B$, for a total price of $\pi_{B}$; hence they obtain a payoff smaller than $U_{B}^{C}+2 M^{\prime}-\pi_{B}^{C *}$. On the other hand, if the consortium is formed, then it can obtain a payoff of $U_{A}^{C}+2 M^{\prime}-\pi_{A}^{C *}$ (which is equal to $U_{B}^{C}+2 M^{\prime}-\pi_{B}^{C *}$ ) from buying bundle $A .^{23}$ Hence $A$ can increase its payoff above $\pi_{A}$.

Lemma 7 Suppose Assumption 1. Under menu pricing, in no equilibrium publisher $j$ obtains a profit strictly higher than the profit $\pi_{j}^{C *}$.

Proof. Suppose that there exists an equilibrium in which publisher $j$ obtains a profit strictly higher than the profit $\pi_{j}^{C *}$. Since $\pi_{j}^{C *}+\pi_{j}^{C *}=2 M^{\prime}$, publisher $k(\neq j)$ obtains a profit strictly lower than $\pi_{j}^{C *}$, which contradicts Lemma 6.

\section{Proof of Proposition 5.}

Lemma 6 and Lemma 7 imply that in any equilibrium each publisher $j$ earns $\pi_{j}^{C *}$. One equilibrium of this game is such that $P_{A}^{C}=\pi_{A}^{C *}, P_{B}^{C}=\pi_{B}^{C *}$, and $p_{A}^{1}=p_{A}^{2}=p_{B}^{1}=$ $p_{B}^{2}=\infty$. Precisely, it is immediate to see that no profitable deviation exists when $\pi_{A}^{C *}=2 M^{\prime}, \pi_{B}^{C *}=0$. If instead $\pi_{A}^{C *}<2 M^{\prime}$, then no profitable deviation for $j$ exists if the consortium is formed because deviation to $P_{j}^{C}>\pi_{j}^{C *}$ induces the consortium to buy only bundle $k(\neq j){ }^{24}$ If publisher $j$ wants to induce the funding agency not to form a consortium, then it needs to offer a payoff higher than $\frac{1}{2}\left(U_{j}^{C}+U_{k}^{C}+2 M^{\prime}\right)$, the payoff the consortium can earn by purchasing bundle $k$ at the price $\pi_{k}^{C *}$. The inequality $U_{j}^{C}+2 M^{\prime}-p_{j}^{1}-p_{j}^{2}>\frac{1}{2}\left(U_{j}^{C}+U_{k}^{C}+2 M^{\prime}\right)$ is equivalent to $p_{j}^{1}+p_{j}^{2}<\frac{1}{2}\left(U_{j}^{C}-U_{k}^{C}+2 M^{\prime}\right)=\pi_{j}^{C *}$, thus again no profitable deviation exists.

\subsection{Proof of Proposition 6}

Let $p_{j}^{i, N C}$ be the equilibrium price and $\pi_{j}^{i, N C}$ the equilibrium profit when there is no consortium. When $\lambda$ is about zero, there exists an equilibrium with the following profile of strategies

$$
\tilde{P}_{j}^{C}=\pi_{j}^{1, N C}+\pi_{j}^{2, N C} \text { for } j=A, B ; \quad \tilde{p}_{j}^{i}=p_{j}^{i, N C} \text { for } i=1,2, j=A, B .
$$

where $\tilde{P}_{j}^{C}$ can be any positive price if $\pi_{j}^{1, N C}+\pi_{j}^{2, N C}=0$.

\footnotetext{
${ }^{23}$ The consortium buys both journals if $P_{B}^{C} \leq \pi_{B}^{C *}$.

${ }^{24}$ Obviously deviation to $P_{j}^{C}<\pi_{j}^{C *}$ is not profitable since it reduces j's profit.
} 
- Suppose that $M \leq U_{A}^{2}-U_{B}^{2}$, which implies that $B$ earns 0 without a consortium. Then $\tilde{P}_{A}^{C}=2 M, \tilde{P}_{B}^{C}>0 ; \tilde{p}_{A}^{1}=M, \tilde{p}_{B}^{1}>0, \tilde{p}_{A}^{2}=M, \tilde{p}_{B}^{2}>0$. Regardless of whether the consortium is formed or not, $A$ earns the maximum feasible profit, and $B$ can do nothing to sell its bundle to $\mathrm{C}$ or to an individual library.

- Suppose that $M>\left|U_{A}^{2}-U_{B}^{2}\right|$. Then $\tilde{P}_{A}^{C}=M+\frac{1}{2}\left(M+U_{A}^{2}-U_{B}^{2}\right), \tilde{P}_{B}^{C}=\frac{1}{2}(M+$ $\left.U_{B}^{2}-U_{A}^{2}\right), \tilde{p}_{A}^{1}=M, \tilde{p}_{B}^{1}>0, \tilde{p}_{A}^{2}=\frac{1}{2}\left(M+U_{A}^{2}-U_{B}^{2}\right), \tilde{p}_{B}^{2}=\frac{1}{2}\left(M+U_{B}^{2}-U_{A}^{2}\right)$. When the transaction cost is zero, the consortium gets formed since in this way it buys both bundles, whereas without a consortium, Library 1 does not buy bundle $B$. Is there any profitable deviation for each publisher?

Since $\tilde{p}_{A}^{1}, \tilde{p}_{B}^{1}, \tilde{p}_{A}^{2}, \tilde{p}_{B}^{2}$ are the equilibrium prices without a consortium, it follows that there is no profitable deviation if the consortium is not formed. Hence, if a profitable deviation exists for $B$, it must be such that the consortium is formed, and pays for bundle $B$ more than $\tilde{P}_{B}^{C}$. But in fact, this is impossible, because $U_{A}^{C}-\tilde{P}_{A}^{C}>U_{B}^{C}-\tilde{P}_{B}^{C}$, and $U_{A}^{C}-\tilde{P}_{A}^{C}>U_{B}^{C}-P_{B}^{C}$ for each $P_{B}^{C}>\tilde{P}_{B}^{C}$. Therefore if $B$ chooses $P_{B}^{C}>\tilde{P}_{B}^{C}$, then the consortium will not be formed or will be formed and buy bundle $A$.

Likewise, if a profitable deviation exist for $A$, it must be such that the consortium is formed, and pays for bundle $A$ more than $\tilde{P}_{A}^{C}$. Hence consider $P_{A}^{C}>\tilde{P}_{A}^{C}$ and notice that if the consortium is not formed, then the libraries in aggregate obtain a payoff of

$$
U_{B}^{2}+2 M-\frac{1}{2}\left(M+U_{B}^{2}-U_{A}^{2}\right)+\left(U_{A}^{1}-p_{A}^{1}\right) x_{A}^{1}+\left(U_{A}^{2}-p_{A}^{2}\right) x_{A}^{2}+\left(U_{B}^{1}-p_{B}^{1}\right) x_{B}^{1},
$$

where $x_{j}^{i}=1$ if library $i$ buys bundle $j$ and $x_{j}^{i}=0$ if library $i$ does not buy it. ${ }^{25}$ Therefore it is necessary that

$$
P_{A}^{C} \leq U_{A}^{C}+\frac{1}{2}\left(M+U_{B}^{2}-U_{A}^{2}\right)-U_{B}^{2}-\left(U_{A}^{1}-p_{A}^{1}\right) x_{A}^{1}-\left(U_{A}^{2}-p_{A}^{2}\right) x_{A}^{2}-\left(U_{B}^{1}-p_{B}^{1}\right) x_{B}^{1} .
$$

Hence, $A$ 's maximum profit is equal to

$$
\lambda\left(U_{A}^{C}+\frac{1}{2}\left(M+U_{B}^{2}-U_{A}^{2}\right)-U_{B}^{2}-U_{A}^{1} x_{A}^{1}-U_{A}^{2} x_{A}^{2}-\left(U_{B}^{1}-p_{B}^{1}\right) x_{B}^{1}\right)+p_{A}^{1} x_{A}^{1}+p_{A}^{2} x_{A}^{2} .
$$

Notice that for $\lambda$ about zero, it is profitable for $A$ to set $p_{A}^{1}=M, p_{A}^{2}=\frac{1}{2}(M+$ $\left.U_{B}^{2}-U_{A}^{2}\right)$, which makes the profit from deviation equal to $\frac{\lambda}{2}\left(M-U_{A}^{2}-U_{B}^{2}\right)+M+$ $\frac{1}{2}\left(M+U_{B}^{2}-U_{A}^{2}\right)$, which is smaller than $M+\frac{1}{2}\left(M+U_{B}^{2}-U_{A}^{2}\right)$ (by Assumption 1$)$, the profit in case of no deviation.

\footnotetext{
${ }^{25}$ Notice that $x_{B}^{2}$ is set equal to 1 because given $p_{B}^{2}=\frac{1}{2}\left(M+U_{B}^{2}-U_{A}^{2}\right)$, library 2 buys bundle $B$ no matter what $p_{A}^{2}$.
} 
- Now suppose that $U_{B}^{2}>U_{A}^{2}$ and $M \leq U_{B}^{2}-U_{A}^{2}$. Then $\tilde{P}_{A}^{C}=M, \tilde{P}_{B}^{C}=M, \tilde{p}_{A}^{1}=M$, $\tilde{p}_{B}^{1}>0, \tilde{p}_{A}^{2}>0, \tilde{p}_{B}^{2}=M$. Hence, if the transaction cost is zero, then the consortium is formed and buys both bundles. As above, there is no profitable deviation if the consortium is not formed. In order for a profitable deviation to exist for $B$, it has to be such that the consortium is formed and buys bundle $B$ for more than $M$, but that cannot occur because $U_{A}^{C}-M>U_{B}^{C}-M$. In order for a profitable deviation to exist for $A$, it has to be such that the consortium is formed and buys $A$ for more than $M$. Hence, consider $P_{A}^{C}>M$ and notice that if the consortium is not formed, then libraries in aggregate obtain a payoff of

$$
\left(U_{A}^{1}-p_{A}^{1}\right) x_{A}^{1}+\left(U_{B}^{1}-p_{B}^{1}\right) x_{B}^{1}+U_{B}^{2}+M .
$$

Therefore it is necessary that

$$
P_{A}^{C} \leq U_{A}^{C}-U_{B}^{2}+M-\left(U_{A}^{1}-p_{A}^{1}\right) x_{A}^{1}-\left(U_{B}^{1}-p_{B}^{1}\right) x_{B}^{1} .
$$

Hence, $A$ 's maximum profit is equal to

$$
\lambda\left(U_{A}^{C}-U_{B}^{2}+M-U_{A}^{1} x_{A}^{1}-\left(U_{B}^{1}-p_{B}^{1}\right) x_{B}^{1}\right)+p_{A}^{1} x_{A}^{1} .
$$

Notice that for $\lambda$ about zero, it is profitable to set $p_{A}^{1}=M$, which makes the profit from the deviation equal to $\lambda\left(U_{A}^{2}-U_{B}^{2}+M\right)+M$, which is not larger than $M$ (since $M \leq U_{B}^{2}-U_{A}^{2}$ ), the profit in case of no deviation.

- Now suppose that $M \geq U_{A}^{1}-U_{B}^{1}$. Then $\tilde{P}_{A}^{C}=\frac{1}{2}\left(2 M+U_{A}^{C}-U_{B}^{C}\right), \tilde{P}_{B}^{C}=\frac{1}{2}(2 M+$ $\left.U_{B}^{C}-U_{A}^{C}\right), \tilde{p}_{A}^{1}=\frac{1}{2}\left(M+U_{A}^{1}-U_{B}^{1}\right), \tilde{p}_{B}^{1}=\frac{1}{2}\left(M+U_{B}^{2}-U_{A}^{2}\right), \tilde{p}_{A}^{2}=\frac{1}{2}\left(M+U_{A}^{2}-U_{B}^{2}\right)$, $\tilde{p}_{B}^{2}=\frac{1}{2}\left(M+U_{B}^{2}-U_{A}^{2}\right)$. As above, there is no profitable deviation if the consortium is not formed, and there is no profitable deviation if it gets formed either, because $U_{A}^{C}-\tilde{P}_{A}^{C}=U_{B}^{C}-\tilde{P}_{B}^{C}$ 


\section{Supporting Information}

\section{Appendix B: Extensions}

\subsection{Complements or Substitutes}

We assume that if library $i$ buys both bundles, then it gets a gross utility of $U_{A}^{i}+U_{B}^{i}+\delta^{i}$, where $\delta^{i}>0$ represents the case of complements and $\delta^{i}<0$ represents the case of substitutes. We assume that the gross utility from consuming both bundles is greater than the gross utility from a single bundle: $U_{A}^{i}+U_{B}^{i}+\delta^{i}>\max \left\{U_{A}^{i}, U_{B}^{i}\right\}$. If a consortium is formed by $n$ libraries and the consortium buys both bundles, the consortium gets a gross utility of $U_{A}^{C}+U_{B}^{C}+\delta^{C}$, where $\delta^{C} \equiv \delta^{1}+\ldots+\delta^{n}$.

We modify our Assumption 1 as follows:

Assumption 1': $M^{i}<U_{A}^{i}+U_{B}^{i}-2\left|\min \left\{0, \delta^{i}\right\}\right|$ for $i=1, \ldots, n$.

If the bundles are complements (i.e., $\delta^{i}>0$ ), Assumption 1' becomes $M^{i}<U_{A}^{i}+U_{B}^{i}$. Hence, when the budget constraint binds $\left(P_{A}^{i}+P_{B}^{i}=M^{i}\right)$, the library gets a strictly positive payoff from buying both bundles. If the bundles are substitutes (i.e., $\delta^{i}<0$ ), then Assumption 1' becomes $M^{i}<U_{A}^{i}+U_{B}^{i}-2\left|\delta^{i}\right|$, and still, when the budget constraint binds, the library gets a strictly positive payoff from buying both bundles.

Let us first consider competition in the market for a given library $i(=1, \ldots, n, C)$ : we eliminate the superscript $i$. Then, under Assumption 1', Lemma 1 extends as follows.

Lemma 8 Consider competition between two publishers in the market for a given library. Without loss of generality, we assume $U_{A} \geq U_{B}$. Suppose that the two bundles can be either complements or substitutes, and $U_{A}+U_{B}+\delta>U_{A}$. Under Assumption 1':

(i) if $M \leq U_{A}-U_{B}$, then publisher $A$ charges $P_{A}=M$, publisher $B$ charges an arbitrary $P_{B}>0$, and the library buys only bundle $A$;

(ii) if $U_{A}-U_{B}<M<U_{A}+U_{B}-2|\min \{0, \delta\}|$, then the publishers charge $P_{A}=$ $\frac{1}{2}\left(M+U_{A}-U_{B}\right), P_{B}=\frac{1}{2}\left(M+U_{B}-U_{A}\right)$, and the library buys both bundles.

Proof. The proof is straightforward and is omitted.

According to the lemma, the introduction of complements or substitutes does not affect equilibrium pricing under Assumption 1'. If the budget is severely limited such that $M \leq U_{A}-U_{B}$ holds, then the library's choice is between buying only bundle $A$ and buying only bundle $B$. Hence, $\delta$ does not affect the pricing. If the budget is not so scarce such that $U_{A}-U_{B}<M<U_{A}+U_{B}-2|\min \{0, \delta\}|$ holds, the equilibrium prices are determined by the binding budget constraint $\left(P_{A}+P_{B}=M\right)$ and the indifference 
condition between buying only bundle $A$ and only bundle $B\left(U_{A}-P_{A}=U_{B}-P_{B}\right)$. Hence, the equilibrium pricing still does not depend on $\delta$. However, in this case, the condition that the library prefers buying both bundles to buying a single bundle should be met. This is trivially met in the case of complements. However, in the case of substitutes $(\delta<0)$, the following inequality should hold

$$
U_{A}+U_{B}-|\delta| \geq U_{j}+M-P_{j}\left(=\frac{1}{2}\left(U_{A}+U_{B}+M\right)\right) \text { for } j=A, B,
$$

which is equivalent to

$$
U_{A}+U_{B}-M \geq 2|\delta| .
$$

Basically, the magnitude of the substitution should be limited, which is met under Assumption 1'.

One consequence of Lemma 8 is that our results on when forming a consortium increases the aggregate payoff of the libraries does not depend on whether the bundles are complements or substitutes as long as $U_{A}^{i}+U_{B}^{i}+\delta^{i}>\max \left\{U_{A}^{i}, U_{B}^{i}\right\}$ and Assumption 1' are satisfied:

Proposition 7 (complements and substitutes) Suppose that for each library $i$, the two bundles can be either complements $\left(\delta^{i}>0\right)$ or substitutes $\left(\delta^{i}<0\right)$. Moreover, suppose that Assumption 1' is satisfied, that $U_{A}^{i}+U_{B}^{i}+\delta^{i}>\max \left\{U_{A}^{i}, U_{B}^{i}\right\}$ for $i=1, \ldots, n$, and that (without loss of generality) $\Delta^{C} \equiv U_{A}^{C}-U_{B}^{C} \geq 0$. Then, whether the consortium increases or reduces the aggregate payoffs of the libraries does not depend on $\delta^{1}, \ldots, \delta^{n}$ : the statement of Proposition 1 obtained when each $\delta^{i}$ is zero still applies when $\delta^{1}, \ldots, \delta^{n}$ are not all zero.

Proof. The proof is straightforward and is omitted.

Essentially, the consortium strictly increases (respectively, reduces) the aggregate payoff of the libraries if it increases (respectively, reduces) the number of bundles consumed for at least one library. The consortium can reduce the aggregate payoff without affecting the number of bundles consumed by each library. This occurs when type $A$ libraries consume only bundle $A$ and type $B$ libraries consume only bundle $B$ whereas the consortium ends up buying only bundle $A$,. However, in this case, $\delta$ does not matter since each library consumes only one journal regardless of whether or not the consortium is formed.

\subsection{Three publishers}

In this subsection, we analyze an extension to three publishers $(A, B, D)$ when there are two libraries with exogenously given budget. Following Jeon and Menicucci (2006, 2011), we introduce an intermediate stage between stage 0 and stage 1 in the timing described in Section 2: 
- Stage 0: The funding agency decides whether to form a consortium or not.

- Stage 0.5: After observing the decision taken in Stage 0, each publisher simultaneously decides whether to be active or not in the market of each library $i(i=1,2, C)$.

- Stage 1: After the decisions taken in Stage 0.5 are observed by all publishers and the libraries (or the consortium), each publisher $j$ active in market $i$ simultaneously chooses $P_{j}^{i}>0$ for $i=1,2$ if the consortium is not formed (for $i=C$ if the consortium is formed).

- Stage 2: Each library (the consortium) decides the bundle(s) to buy.

If a publisher is not active in the market of library $i$, it does not offer any bundle to the library and therefore the library cannot buy its bundle. As in Jeon and Menicucci (2006, 2011), for equilibrium selection, we assume that at Stage 0.5, a publisher decides not to be active in the market of library $i$ if it expects to make zero profit in that market. The assumption can be justified if a publisher should incur a very small but positive cost of contracting a library. Without this assumption, the prices of the bundles that library $i$ buys may depend on the prices of the bundles library $i$ does not buy, which creates multiple equilibria.

Consider competition in the market for a given library $i(=1,2, C)$. We eliminate the superscript $i$ and, without loss of generality, assume $U_{A} \geq U_{B} \geq U_{D}>0$. Then, from our previous papers, we have

Lemma 9 (Jeon and Menicucci, 2006 and 2011) Consider competition between three publishers in the market for a given library (or consortium), which starts from Stage 0.5. Then, there exists a unique subgame perfect Nash equilibrium. The equilibrium active publishers and prices are given by:

(i) if $M \leq U_{A}-U_{B}$, then only publisher $A$ is active, and charges $P_{A}=M$;

(ii) if $U_{A}-U_{B}<M \leq U_{A}+U_{B}-2 U_{D}$, then only publishers $A$ and $B$ are active, and charge $P_{A}=\frac{1}{2}\left(M+U_{A}-U_{B}\right), P_{B}=\frac{1}{2}\left(M+U_{B}-U_{A}\right)$;

(iii) if $M>U_{A}+U_{B}-2 U_{D}$, then all three publishers are active, and each publisher $j$ $(=A, B, D)$ charges $P_{j}=U_{j}-\frac{1}{3} \max \left\{0, U_{A}+U_{B}+U_{D}-M\right\}$.

As the budget increases, the number of active publishers increases. Note that for $M \leq U_{A}+U_{B}+U_{D}$, the budget is exhausted in the equilibrium.

In what follows, we compare the outcome under the consortium with the one without the consortium. Let $U_{(r)}^{i}$ represent the surplus that library $i(=1,2, C)$ obtains from its $r$-th best $(r=1,2,3)$ bundle: the ranking is specific to $i$. We assume:

Assumption 1": $U_{A}^{i}+U_{B}^{i}+U_{D}^{i} \geq M^{i}>0$, for $i=1,2$. 
Assumption 1" means that the budget constraint binds for each library and also implies $U_{(1)}^{C}+U_{(2)}^{C}+U_{(3)}^{C} \geq M^{C}$. Arguing as in Proposition 1, we obtain

Proposition 8 Suppose there are three publishers, that Assumption 1" is satisfied, and that (without loss of generality) $U_{(1)}^{C}=U_{A}^{C}$.

(i) If $M^{C} \leq U_{(1)}^{C}-U_{(2)}^{C}$, the consortium buys only bundle $A$. The consortium at least weakly reduces the aggregate payoff of the libraries and strictly reduces it unless each library buys only bundle A without the consortium.

(ii) If $M^{C}>\left[U_{(1)}^{C}-U_{(3)}^{C}\right]+\left[U_{(2)}^{C}-U_{(3)}^{C}\right]$, the consortium buys all the three bundles. The consortium at least weakly increases the aggregate payoff of the libraries and strictly increases it unless each library buys all three bundles without the consortium.

Proof. It is straightforward from Lemma 9 and is omitted.

This result makes clear the effect of consortium in two extreme cases: when the consortium strongly prefers one bundle over the other two bundles, or the consortium has sufficiently balanced preferences such that its utility from the two best bundles is not too higher than twice the utility from the worst bundle. In the first case, each library is strictly worse off under the consortium, unless each library buys only bundle $A$ without the consortium. In the second case, each library is strictly better off under the consortium, unless each library buys all three bundles without the consortium.

The proposition does not consider the intermediate case in which $U_{(1)}^{C}-U_{(2)}^{C}<M^{C} \leq$ $U_{(1)}^{C}+U_{(2)}^{C}-2 U_{(3)}^{C}$, because under these inequalities the consortium buys its two preferred bundles only, and then establishing the effect on the aggregate payoff of the libraries requires additional assumptions on preferences. Even for the two extreme cases, it is not obvious to see when $M^{C} \leq U_{(1)}^{C}-U_{(2)}^{C}$ or $M^{C}>\left[U_{(1)}^{C}-U_{(3)}^{C}\right]+\left[U_{(2)}^{C}-U_{(3)}^{C}\right]$ holds. In what follows, we introduce additional assumptions which allow us to make comparison in a simple two-dimensional space of $(\rho, \Delta)$ as we have done for the case of two publishers (see Figure 1). This requires us to consider a specific class of parameter values. More precisely, we assume:

Assumption 3: $M^{1}=M^{2}=M, U_{(1)}^{i}-U_{(2)}^{i}=U_{(2)}^{i}-U_{(3)}^{i}$, for $i=1,2$.

Assumptions 3 means that each library has the same budget and that the surplus gap between two bundles of adjacent ranking is constant for each library. Concerning the ranking of the bundles for Library 1, without loss of generality, we can assume $U_{(1)}^{1}=U_{A}^{1}$, $U_{(2)}^{1}=U_{B}^{1}, U_{(3)}^{1}=U_{D}^{1}$. Regarding the ranking of the bundles for Library 2, we consider two possibilities: either Library 2 has the same ranking as Library 1 (i.e., $U_{(1)}^{2}=U_{A}^{2}, U_{(2)}^{2}=U_{B}^{2}$, $U_{(3)}^{2}=U_{D}^{2}$ ) or has the opposite ranking (i.e., $U_{(1)}^{2}=U_{D}^{2}, U_{(2)}^{2}=U_{B}^{2}, U_{(3)}^{2}=U_{A}^{2}$ ). Without loss of generality, we can also assume $U_{(1)}^{1}-U_{(2)}^{1} \geq U_{(1)}^{2}-U_{(2)}^{2}$. Let $U_{(1)}^{1}-U_{(2)}^{1} \equiv \Delta>0$. 
Then, we can write $U_{(1)}^{2}-U_{(2)}^{2}=|\rho| \Delta$ with $|\rho| \in[0,1]$, where $\rho>0$ if both libraries have the same ranking, and $\rho<0$ if they have opposite ranking. Summarizing, we have:

$$
\begin{gathered}
U_{A}^{1}=U_{B}^{1}+\Delta, U_{D}^{1}=U_{B}^{1}-\Delta ; \\
U_{A}^{2}=U_{B}^{2}+\rho \Delta, U_{D}^{2}=U_{B}^{2}-\rho \Delta \text { if } \rho \geq 0 ; \\
U_{A}^{2}=U_{B}^{2}-|\rho| \Delta, U_{D}^{2}=U_{B}^{2}+|\rho| \Delta \text { if } \rho<0 .
\end{gathered}
$$

This implies also that the consortium has the same ranking as Library 1 regardless of the sign and value of $\rho \in[-1,1]: U_{(1)}^{C}=U_{A}^{C}, U_{(2)}^{C}=U_{B}^{C}, U_{(3)}^{C}=U_{D}^{C}$.

In addition, we can normalize the budget by considering $M^{\prime}=M / \Delta$. Then, from Lemma 9, we have:

Corollary 3 Consider competition between three publishers in the market for a given library (or consortium). Suppose Assumptions 1" and 3.

(i) In the market for Library 1, the library buys only bundle $A$ if $M^{\prime} \leq 1$, only bundles $A$ and $B$ if $1<M^{\prime} \leq 3$, all bundles if $M^{\prime}>3$.

(ii) In the market for Library 2;

(a) when $\rho>0$, the library buys only bundle $A$ if $M^{\prime} \leq \rho$, only bundles $A$ and $B$ if $\rho<M^{\prime} \leq 3 \rho$, all bundles if $M^{\prime}>3 \rho$;

(b) when $\rho=0$, it buys all bundles;

(c) when $\rho<0$, it buys only bundle $D$ if $M^{\prime} \leq|\rho|$, only bundles $B$ and $D$ if $|\rho|<M^{\prime} \leq$ $3|\rho|$, all bundles if $M^{\prime}>3|\rho|$.

(iii) In the market for the library consortium, the consortium buys only bundle $A$ if $M^{\prime} \leq(1+\rho) / 2$, only bundles $A$ and $B$ if $(1+\rho) / 2<M^{\prime} \leq 3(1+\rho) / 2$, all bundles if $M^{\prime}>3(1+\rho) / 2$.

Note first that if $M^{\prime}>3$, both libraries consume all three bundles regardless of whether or not the consortium is formed. Therefore, we focus on $M^{\prime} \leq 3$ for the comparison. However, when the consortium ends up consuming only two bundles, even under Assumptions 1 " and 3, many cases arise depending on the values of $\left(M^{\prime}, \rho\right)$. In order to further reduce the number of cases, we assume:

Assumption 4: $U_{(2)}^{1}=U_{(2)}^{2}, U_{(1)}^{1}<U_{(2)}^{1}+U_{(3)}^{1}$.

Under Assumption 3 and 4, the surplus that one library obtains from its first-best bundle (respectively, its second-best bundle) is higher than the surplus that the other library obtains from its second-best bundle (respectively, its third-best bundle) and any library obtains a higher surplus from consuming its second-best and third-best bundles than from consuming its first-best bundle only. 
Our results in this case are described in Figure 4, in which the lower bold line with a positive slope describes the cutoff values of $M^{\prime}$ for the consortium to consume two bundles instead of one and the upper bold line with a positive slope describes the cutoff values of $M^{\prime}$ for the consortium to consume three bundles instead of two. Similarly, the two horizontal dash lines describe the cutoff values for Library 1 and the two dash lines (with a positive slope for $\rho>0$ and with a negative slope for $\rho<0$ ) describe the cutoff values for Library 2. For each area generated by these lines, we indicate whether the consortium increases or reduces or has no effect on the aggregate payoff of the libraries by using + or - or 0. Next proposition summarizes our results:

Proposition 9 (exogenous budget and correlation) Consider that the funding agency can form a consortium of two libraries when three publishers compete. Suppose Assumption $1 ", 3,4$ and $M^{\prime} \leq 3$.

(i) (opposite preferences) When $\rho<0$,

(a) the intervals of $M^{\prime}$ in which the consortium strictly increases the aggregate payoff of the libraries expands with $|\rho|$ such that the consortium strictly increases it for any $M^{\prime} \leq 3$ when $|\rho|=1$;

(b) the intervals of $M^{\prime}$ in which the consortium strictly decreases the aggregate payoff of the libraries shrinks with $|\rho|$.

(ii) (similar preferences) When $\rho>0$,

(a) the intervals of $M^{\prime}$ in which the consortium strictly increases the aggregate payoff of the libraries shrinks with $\rho$ such that the consortium has no effect on it for any $M^{\prime} \leq 3$ when $\rho=1$;

(b) the sum of the lengths of the intervals of $M^{\prime}$ in which the consortium strictly decreases the aggregate payoff of the libraries increases with $\rho$ up to $\rho=1 / 3$ and then decreases with $\rho$ such that the consortium has no effect on it for any $M^{\prime} \leq 3$ when $\rho=1$.

Proof. The proof is straightforward and is omitted.

We notice that the results of Figure 4 and Proposition 9 are remarkably similar to those obtained from two publishers in Figure 1 and Proposition 2. 


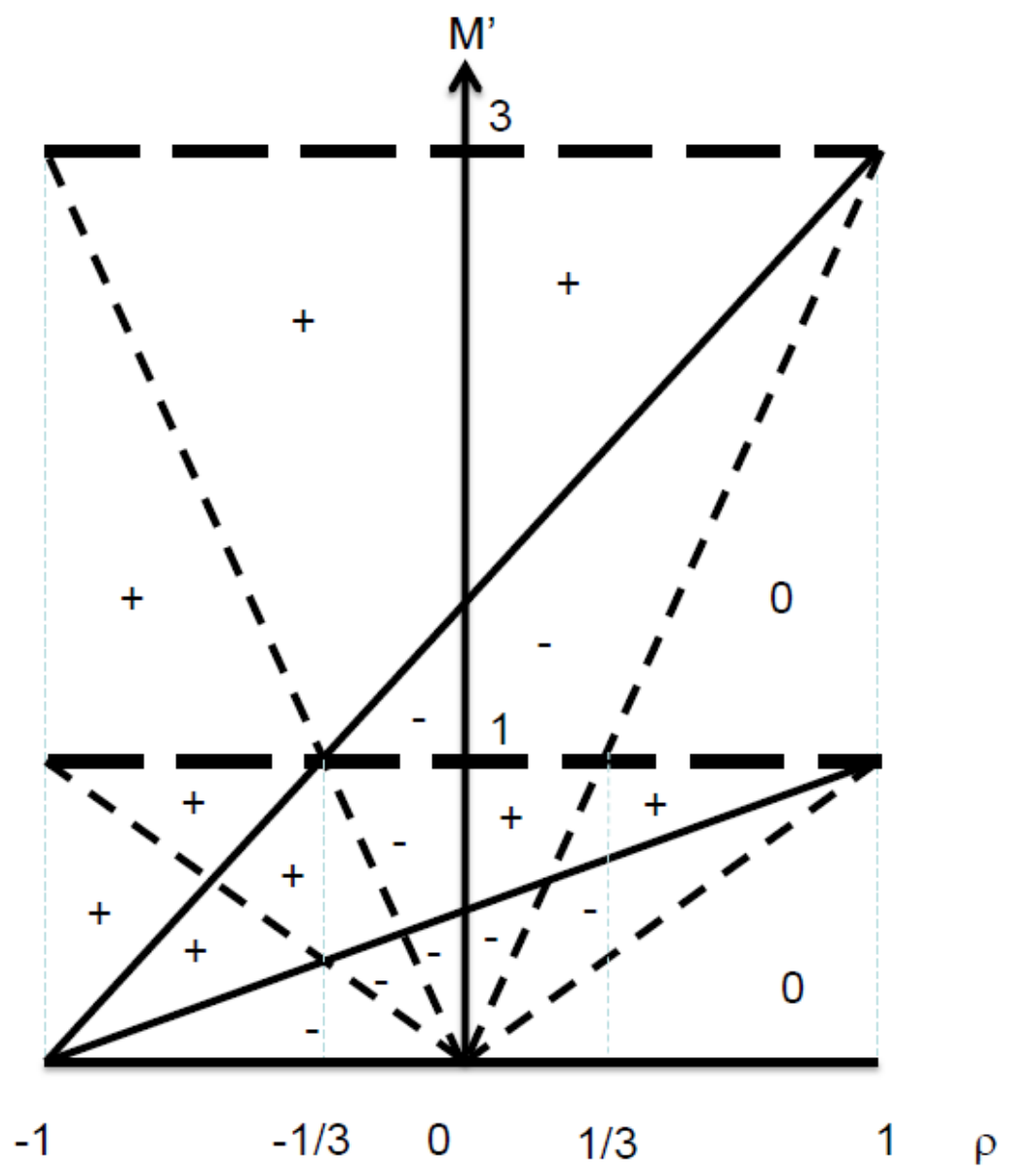

Figure 4: Effect of consortium on the aggregate payoff of the libraries when three publishers compete 